\title{
Cytoplasmic travels of the ecdysteroid receptor in target cells: pathways for both genomic and non-genomic actions
}

\section{Xanthe Vafopoulou * and Colin G. H. Steel}

Biology Department, York University, Toronto, ON, Canada

\section{Edited by:}

Joe Hull, USDA Agricultural Research

Service, USA

\section{Reviewed by:}

Kirst King-Jones, University of

Alberta, Canada

Chantal Dauphin-Villemant, CNRS,

France

\section{*Correspondence:}

Xanthe Vafopoulou, Biology

Department, York University, 4700

Keele Street, Toronto, ON, Canada

M3J 1 P3.

e-mail:xanthev@yorku.ca
Signal transduction of the insect steroid hormones, ecdysteroids, is mediated by the ecdysteroid receptor, EcR. In various cells of the insect Rhodnius prolixus, EcR is present in both the nucleus and the cytoplasm, where it undergoes daily cycling in abundance and cellular location at particular developmental times of the last larval instar that are specific to different cell types. EcR favors a cytoplasmic location in the day and a nuclear location in the night. This study is the first to examine the potential mechanisms of intracellular transport of EcR and reveals close similarities with some of its mammalian counterparts. In double and triple labels using several antibodies, immunohistochemistry, and confocal laser scanning microscopy, we observed co-localization of EcR with the microtubules (MTs). Treatments with either the MT-stabilizing agent taxol or with colchicine, which depolymerizes MTs, resulted in considerable reduction in nuclear EcR with a concomitant increase in cytoplasmic EcR suggesting that MT disruption inhibits receptor accumulation in the nucleus. EcR also co-localizes with the chaperone Hsp90, the immunophilin FKBP52, and the light chain 1 of the motor protein dynein. All these factors also co-localize with MTs. We propose that in Rhodnius, EcR exerts its genomic effects by forming a complex with Hsp90 and FKBP52, which uses dynein on MTs as a mechanism for daily nucleocytoplasmic shuttling. The complex is transported intact to the nucleus and dissociates within it. We propose that EcR utilizes the cytoskeletal tracks for movement in a manner closely similar to that used by the glucocorticoid receptor. We also observed co-localization of EcR with mitochondria which suggests that $\mathrm{ECR}_{\mathrm{C}}$, like its mammalian counterparts, may be involved in the coordination of non-genomic responses of ecdysteroids in mitochondria.

Keywords: microtubules, Hsp90, immunophilin, dynein, nucleocytoplasmic receptor shuttling, mitochondria, steroid hormone, Rhodnius

\section{INTRODUCTION}

Ecdysteroids, the steroid hormones of insects, coordinate a wide variety of developmental and physiological processes by binding to a specific cellular receptor protein called the ecdysteroid receptor $(\mathrm{EcR})$ and regulate tissue- and stage-specific gene transcription. $\mathrm{EcR}$ is a ligand-activated transcription factor and a member of the steroid receptor superfamily (reviewed by Henrich, 2009). The functional ecdysone receptor in insects is a heterodimer of the EcR protein and a RXR homolog, Ultraspiracle (Usp; review by Spindler et al., 2009). EcR and Usp and their isoforms have been cloned from many insects and other arthropods and their differential expression is considered a major factor in regulation of the diversity of cellular responses to ecdysteroids during development (review by Riddiford et al., 2003). There is an extensive literature on the molecular structure, mechanisms of partnership with Usp and transcriptional activity of EcR (e.g., reviews by Henrich, 2009; Nakagawa and Henrich, 2009; Spindler et al., 2009). In mammals, steroid receptors and most of the nuclear factors involved in signaling cascades are continually shuttling between the nucleus and the cytoplasm (review by Echeverria and Picard, 2010). EcR possesses both nuclear localization and nuclear export signals (Gwózdz et al., 2007) and interacts with exportin 1 (Betanska et al., 2011). The presence of cytoplasmic EcR in insects has been noted but no significance has been attributed to it (Lammerding-Köppel et al., 1998; Gwózdz et al., 2007; Nieva et al., 2007; Betanska et al., 2011). The only evidence that EcR undergoes shuttling derives from evidence in Rhodnius prolixus (Vafopoulou and Steel, 2006). More recently, it was found that the orphan nuclear receptor DHR4 also undergoes nucleocytoplasmic shuttling in prothoracic gland (PG) cells of Drosophila (Ou et al., 2012) suggesting that shuttling of nuclear receptors may be a common phenomenon in insects.

Rhodnius is a historically favored model system for studies of the hormonal control of insect development (Wigglesworth, 1985). A blood meal is required by each stage to initiate development to the next stage, which results in remarkable synchrony in a group of insects fed simultaneously throughout the 21-days of larval-adult development. This synchrony results in unmatched precision of timing of developmental events. It has also enabled this laboratory to identify the first circadian rhythms in insect hormones, particularly in the levels of the steroid molting hormones (ecdysteroids; Ampleford and Steel, 1985; Vafopoulou and Steel, 1991; review by Steel and Vafopoulou, 2006). Recently we also showed that EcR shuttles between nucleus and cytoplasm of several cell types and that this shuttling occurs with a circadian rhythm. 
The abundance of EcR in the nuclei peaks during the night and decays during the day, whereas the abundance in the cytoplasm shows an inverse trend and peaks during the day and reaches low levels in the night. This shuttling rhythm persists in continuous darkness, emphasizing its circadian nature (Vafopoulou and Steel, 2006). The circadian rhythm of shuttling is in synchrony with the circadian rhythm of circulating ecdysteroids (Vafopoulou and Steel, 2006; Vafopoulou, 2009). Circadian shuttling had not been seen previously in any animal. The present paper confirms these initial findings but also shows that shuttling can be restricted to a period of only a few of the 21-days of development in certain cell types, but occurs on every day of development in others.

The mechanisms underlying EcR transport within cells has never been studied. We proposed recently, using double immunolabels, that EcR may utilize molecular machinery involving cytoskeletal tracks, similar to vesicle transport (Vafopoulou, 2009). The cytoskeleton has been implicated as a system for targeted movement of several mammalian steroid receptors. The primary objective of the present article is to investigate the relationship between EcR in the cytoplasm and the motor machinery of microtubules (MTs) and mitochondria in several cell types of Rhodnius. We report that EcR in the cytoplasm becomes co-localized with both the chaperone Hsp90 and co-chaperone immunophilin FKBP52 and also with the light chain 1 of the motor protein dynein of microtubules (MTs) and tubulin of MTs, indicating that EcR may form a complex with Hsp90 and FKBP52, similar to that of its mammalian counterparts. This complex may use dynein as the motor protein on MTs for targeted movement to the nucleus. This movement system is very similar to that utilized by the glucocorticoid receptor (GR). We also found that the inferred EcR-Hsp-FKBP52 complex shuttles intact into the nucleus and that EcR dissociates from the chaperones inside the nucleus. These findings bring EcR into closer alignment with the more thoroughly analyzed mammalian steroid hormones.

A second objective of the present work was to examine relationships between EcR and cytoplasmic organelles, to identify potential non-genomic roles for cytoplasmic EcR. Many examples of rapid actions of ecdysteroids cannot be explained by genomic actions and imply potential non-genomic actions of ecdysteroids (reviewed by Schlattner et al., 2006), which indicate a functional significance for the presence of EcR in the cytoplasm. Many actions of ecdysteroids are rapid and transient and usually involve electrochemical changes at the cell membrane (review by Schlattner et al., 2006). Membrane-bound molecules that appear to be related to conventional EcR have been reported (Elmogy et al., 2004, 2007). We reported recently that EcR associates with mitochondria in the cytoplasm of PG cells of Rhodnius (Vafopoulou, 2009). Here, we report co-localization of cytoplasmic EcR with mitochondria in all cell types studied, showing that the mitochondria are a potential site for non-genomic actions of ecdysteroids.

\section{MATERIALS AND METHODS}

\section{ANIMALS}

Fifth (last) larval instar males of $R$. prolixus were raised at $28^{\circ} \mathrm{C}$ in a 12-h light:12 h dark regime. Animals exist in a state of arrested development until given a blood meal, which initiates larval-adult development. The day of feeding was designated day 0. Days of development were counted from the day of feeding. Ecdysis is gated with a median on day 21 . Tissues were dissected at day 13 after a blood meal.

\section{ANTIBODIES, PROTEINS, AND REAGENTS}

A mouse monoclonal EcR antibody (9B9) was purchased from Developmental Studies Hybridoma Bank (University of Iowa). This antibody detects an epitope between residues 127 and 354 of Manduca sexta EcR; these sequences are present in all EcR isoforms of Manduca, hence they were designated EcR-common (Fujiwara et al., 1995; Jindra et al., 1996). This antibody has been used previously to localize EcR in PG cells (Vafopoulou, 2009). Several lines of evidence validated the specificity of this antibody in recognizing the EcR of Rhodnius. First, this antibody recognizes only three proteins in Rhodnius PGs on Western blots at 79,64 , and $56 \mathrm{kDa}$ (Vafopoulou, 2009). Proteins of closely similar molecular masses to those above were also identified in various Rhodnius tissue extracts using two other antibodies (15C3 and $10 \mathrm{~F} 1$ ) which were also produced against the common region of Manduca EcR (Vafopoulou et al., 2005). These three immunoreactive proteins possess very similar molecular masses to EcR isoforms reported in other insects and have been considered to represent the isoforms of Rhodnius EcR (references in Vafopoulou et al., 2005; Vafopoulou, 2009). Upregulation of EcR was seen with all three antibodies in response to an increase in ecdysteroid levels in vivo and in vitro (Vafopoulou et al., 2005; Vafopoulou, 2009), as is expected for ligand inducible receptors such as EcR (Henrich, 2009). The 9B9 antibody was used immunohistochemically to demonstrate a circadian rhythm in abundance and cytoplasmic location of EcR in PGs (Vafopoulou, 2009). This circadian rhythm was of the same phase and period length as had been reported earlier using the other two EcR antibodies (Vafopoulou and Steel, 2006). We conclude that all three antibodies recognize the native Rhodnius EcR. This antibody was used at a 1:1000 dilution.

A rabbit polyclonal antibody against the whole human Hsp90 was purchased from Stressgen (Enzo Life Science International, Plymouth Meeting, PA, USA) and was used at a 1:250 dilution. This antibody recognizes a protein of about $90 \mathrm{kDa}$ in samples from many eukaryotes, including Drosophila (supplier data). Blast searches revealed that Hsp90 is a highly conserved protein among animals; it displays a $60-80 \%$ amino acid identity between a variety of mammals and insects. The nucleotide sequences of the Hsp90 genes of insects are highly conserved showing 80-99\% identities (see references in Zhang and Denlinger, 2010; Shu et al., 2011). A Blast search of the Rhodnius trace archive revealed two sequential, long stretches (total of about 1000 nucleic acids) that share about $73 \%$ identity with the Hsp90 genes of humans and insects.

One class of Hsp90-binding co-chaperones is the FK506 immunophilin FKBP52. A rabbit polyclonal antibody against amino acids $1-50$ at the amino end of human FKBP52 was purchased from Novus Biologicals (Littleton, CO, USA) and was used at a dilution of 1:100. A Blast search revealed that human FKBP52 shares about 50\% amino acid identity with its insect homologs; the amino acid identity in the 1-50 sequence ranges between 30 and $63 \%$ between humans and several insect species like Drosophila, Bombyx, and Apis. A Blast search of the Rhodnius 
trace archive revealed two sequential, highly conserved regions that share $85-100 \%$ identity with the coding sequence of the 50 amino acids at the amino end of human FKBP52.

A rabbit polyclonal antibody (DNLL1/2) against the whole molecule (89 amino acids) of human dynein light chain I (DNLL1) was purchased from Santa Cruz Biotechnology (Santa Cruz, CA, USA) and was used at dilution of 1:100. This antibody also recognizes the closely similar DYNLL2 in humans. DYNLL1 is highly conserved across species. For example, the amino acid sequence of Drosophila DYNLL1 shares about 92\% similarity with the DYNLL1 of humans, Chlamydomonas reinhardtii, and Caenorhabditis elegans (Dick et al., 1996). A Blast search of the Rhodnius trace archive revealed five sequential regions that share 78-93\% identity with the coding sequences of human DYNLL1.

Native human Hsp90 (Enzo Life Science International, Plymouth Meeting, PA, USA), full-length, recombinant, His-tagged FKBP52 (Novus Biologicals, Littleton, CO, USA), and lysate of human DYNLL1 from transfected 293 T cells (Santa Cruz Biotechnology, Santa Cruz, CA, USA) were used as positive controls on Western blots. Each of these proteins produce single bands when they immunoreact with their respective antibodies listed above (manufacturers' data sheets); their respective migration positions correspond to approximately $90 \mathrm{kDa}$ for Hsp90, $54 \mathrm{kDa}$ for FKBP52, and $10 \mathrm{kDa}$ for DYNLL1.

MTs were labeled with a rat monoclonal antibody against tyrosylated $\alpha$-tubulin (YL 1/2) which was purchased from AbD Serotec (Raleigh, NC, USA) and was used at a 1:400 dilution. This antibody specifically recognizes a linear sequence with an aromatic residue at the C-terminus, with two adjacent, negatively charged, amino acids; this sequence is represented by Gly-Gly-Tyr in tyrosylated $\alpha$-tubulin (manufacturer's information).

Mitochondria were labeled with a cell-permeant, mitochondriaselective fluorescent dye, MitoTracker Deep Red 633, which has been used before to localize mitochondria in Rhodnius PGs (Vafopoulou, 2009). Acidic organelles, including lysosomes, were labeled with a cell-permeant, fluorescent dye LysoTracker Red DND-99. Both dyes were purchased from Molecular Probes (Eugene, OR, USA) and used according to manufacturer's instructions.

For single and double label immunohistochemistry the following fluorescent secondary antibodies were used: an Alexa fluor 488 (green) goat anti-mouse IgG was used to localize EcR; an Alexa fluor 555 (red) goat anti-rat IgG was used to localize tubulin; an Alexa fluor 555 (red) goat anti-rabbit IgG was used to localize Hsp90 and FKBP52. For triple label experiments, an Alexa fluor 488 (green) goat anti-mouse IgG was used to localize EcR, an Alexa fluor 555 (red) goat anti-rat IgG was used to localize tubulin and an Alexa fluor 633 (far red) goat anti-rabbit IgG was used to localize DNLL1. All secondary antibodies were purchased from Molecular Probes (Eugene, OR, USA) and were used at 1:200 dilutions.

Taxol (paclitaxel) and colchicine were purchased from SigmaAldrich (St. Louis, MO, USA). Vectashield mounting medium was purchased from Vector Laboratories (Burlingame, CA, USA). PVDF filters were purchased from Millipore (Billerica, MA, USA). Ten percent SDS-PAGE gels, 15\% Tris-Tricine gels, and kaleidoscope pre-stained standards were purchased from Bio-Rad (Hercules, CA, USA). Amicon Ultra- $0.5 \mathrm{ml}$ (Ultracel-PL membrane) centrifugal tubes for protein purification and concentration were purchased from Millipore (Billerica, MA, USA). Four types of tubes were used for protein filtration with molecular sizes cut-off at $10,30,50$, and $100 \mathrm{kDa}$.

\section{IMMUNOHISTOCHEMISTRY AND IMAGE COLLECTION}

Tissues were fixed according to an established protocol (Vafopoulou, 2009). In order to preserve the cytoskeleton and have good visualization of nuclear and cytoplasmic EcR, tissues were dissected in PBS ( $\mathrm{pH}$ 7.4) containing $10 \mathrm{mM}$ EGTA and $1 \mathrm{mM} \mathrm{MgSO}$; MT assembly was induced by decreasing $\mathrm{Ca}^{++}$ concentration by addition of the chelating agent EGTA. In single label experiments with anti-EcR (to demonstrate the presence of cytoplasmic EcR), tissues were incubated in $\mathrm{PBS}$ at $37^{\circ} \mathrm{C}$ for $30 \mathrm{~min}$ to allow MTs to polymerize. In double label experiments, co-localization of EcR with MTs or mitochondria was visualized in tissues where MTs were stabilized in live cells with $10 \mu \mathrm{M}$ taxol in PBS for $1 \mathrm{~h}$ at $37^{\circ} \mathrm{C}$, prior to fixation. Taxol was obtained from a $10-\mathrm{mM}$ stock solution in dimethyl sulfoxide (DMSO). DMSO was added to aid dissolution of taxol. Likewise, in order to ascertain co-localization of anti-EcR with mitochondria, the taxol-treated tissues were incubated for $45 \mathrm{~min}$ at $37^{\circ} \mathrm{C}$ in $400 \mathrm{nM}$ MitoTracker Deep Red 633, a cell-permeant fluorescent probe specific for mitochondria. This probe was well retained following cell fixation. In order to ascertain co-localization of $\mathrm{EcR}$ with acidic organelles (like lysosomes), tissues were incubated for $45 \mathrm{~min}$ at $37^{\circ} \mathrm{C}$ in $50 \mathrm{nM}$ LysoTracker Red DND-99. All tissues were then fixed in freshly prepared $4 \%$ paraformaldehyde containing $0.15 \%$ glutaraldehyde $\left(2 \mathrm{~h}\right.$ at $\left.37^{\circ} \mathrm{C}\right)$. Addition of glutaraldehyde to the fixative enhances preservation of MTs by cross-linking them inside the cell. The tissues were washed thoroughly in PBS and then pre-incubated in $5 \%$ control serum containing $1 \%$ Triton X100 as a permeabilizing agent followed by secondary antibody incubation ( $2 \mathrm{~h}$ at room temperature). Tissues were mounted in Vectashield.

The pattern of co-localization of EcR with MTs was compared in tissues where MTs were depolymerized with colchicine. Depolymerization was achieved by incubating live cells with $10 \mu \mathrm{M}$ colchicine in PBS for $1 \mathrm{~h}$ at room temperature. For controls, the primary antibodies were replaced with non-immune serum or the secondary antibodies were replaced with PBS, or MitoTracker Deep Red 633 was omitted. Fluorescence levels in controls were indistinguishable from the background and autofluorescence was not detected. Fluorescence resulting from antibody binding to cells is called here EcR, HsP90, FKBP52, DYNLL1, or tubulin fluorescence depending on the antibody. Ten animals were dissected at each indicated time point.

Digital optical sections at $1 \mu \mathrm{m}$ distances were viewed with an Olympus FV300 confocal laser scanning microscope. Red and green fluorescence were scanned using separate channels to excite Alexa fluor 488 at $488 \mathrm{~nm}$, Alexa fluor 555 at $568 \mathrm{~nm}$, and MitoTracker Deep Red 633 and Alexa fluor 633 at $644 \mathrm{~nm}$. Stringent use of filter blocks was employed to prevent bleed-through between channels. Settings were kept identical for all scanning sessions. Images were processed using Image J 1.41 (NIH) and Adobe Photoshop CS5. Images were modified only to adjust contrast and merge files. 


\section{SDS-PAGE AND WESTERN BLOTS}

To confirm the specificities of the antibodies against proteins related to human Hsp90, FKBP52, and DYNLL1, protein samples from day 13 salivary glands (SGs) were immunoblotted. Batches of 30 SGs each were homogenized by sonication in lysis buffer (50 mM Tris-HCl pH 7.4; 150 mM NaCl, 1\% Triton X-100, $1 \mathrm{mM}$ EDTA, $1 \mathrm{mM}$ dithiothreitol (DTT), $1 \mathrm{mM}$ phenylmethyl sulfonylfluoride (PMSF), $2 \mu \mathrm{g} / \mathrm{ml}$ leupeptin, and $1 \mathrm{mg} / \mathrm{ml}$ each antipain, aprotinin, and pepstatin $\mathrm{A}$ and centrifuged at 15,000 $\times g$ for $10 \mathrm{~min}$ at $4^{\circ} \mathrm{C}$ to remove cell debris. The supernatant was retrieved and fractioned into different molecular size fractions using Amicon Ultra centrifugation tubes. In order to concentrate and partially isolate the Hsp90-related proteins from other proteins, supernatants were centrifuged using a $100-\mathrm{kDa}$ filter membrane. The retentate was discarded to remove proteins $>100 \mathrm{kDa}$ and the filtrate was re-centrifuged using a $50-\mathrm{kDa}$ filter membrane to remove proteins $<50 \mathrm{kDa}$. Then the retentate, which contained proteins in the range of $50-100 \mathrm{kDa}$ was used on Western blots. For FKBP52, the supernatant was first centrifuged with a $50-\mathrm{kDa}$ filter membrane to remove proteins $>50 \mathrm{kDa}$ and the filtrate was re-centrifuged using a $10-\mathrm{kDa}$ filter membrane. The retentate, which contained proteins in the range of $10-50 \mathrm{kDa}$ was used on Western blots. For DYNLL1, the supernatant was centrifuged using a $30-\mathrm{kDa}$ filter membrane to remove all proteins $>30 \mathrm{kDa}$ and the filtrate was used on Western blots. $10 \%$ SDS-PAGE was used for identifying Hsp90- and FKBP52-related proteins and 15\% Tris-Tricine PAGE was used for identification of DYNLL1-related proteins. Western blots were performed using standard procedures. Proteins were electrophoretically transferred onto PVDF filters. Signal detection on filters was performed using the $O$-dianisidine reaction. As positive controls, purified proteins for human Hsp90, FKBP52, and DYNLL1 were used.

The Western blots in Figure 1 show that Rhodnius contains proteins that immunoreact with the antibodies against Hsp90 (left blot), FkBP52 (middle blot), and DYNLL1 (right blot). Two immunoreactive protein bands were revealed with anti-Hsp90, a major one at approximately $90 \mathrm{kDa}$ and a minor one at approximately $85 \mathrm{kDa}$. The major $90 \mathrm{kDa}$ immunoreactive band comigrated with the single control human Hsp90 band. A single immunoreactive protein band was revealed with anti-FKBP52 that co-migrated with the single control human FKBP52 band at approximately $45 \mathrm{kDa}$. A single immunoreactive protein band was revealed with anti-DYNLL1 that co-migrated with the single control human DYNLL1 band at approximately $15 \mathrm{kDa}$. Various gels were prepared using the unused fractions from these fractionations, but were found to contain no bands that were immunoreactive with the relevant antibody. Therefore, the SG cells of Rhodnius contain proteins electrophoretically and immunologically related to human Hsp90, FKBP90, and DYNLL1.

\section{RESULTS}

\section{CYCLING OF ECR IN RHODNIUS CELLS}

We reported before the presence of a circadian rhythm in abundance and subcellular localization in EcR in several different cell types using a different EcR antibody (Vafopoulou and Steel, 2006) and a clear daily rhythm in PG cells using the 9B9 antibody (Vafopoulou, 2009). Presently, we confirmed the presence

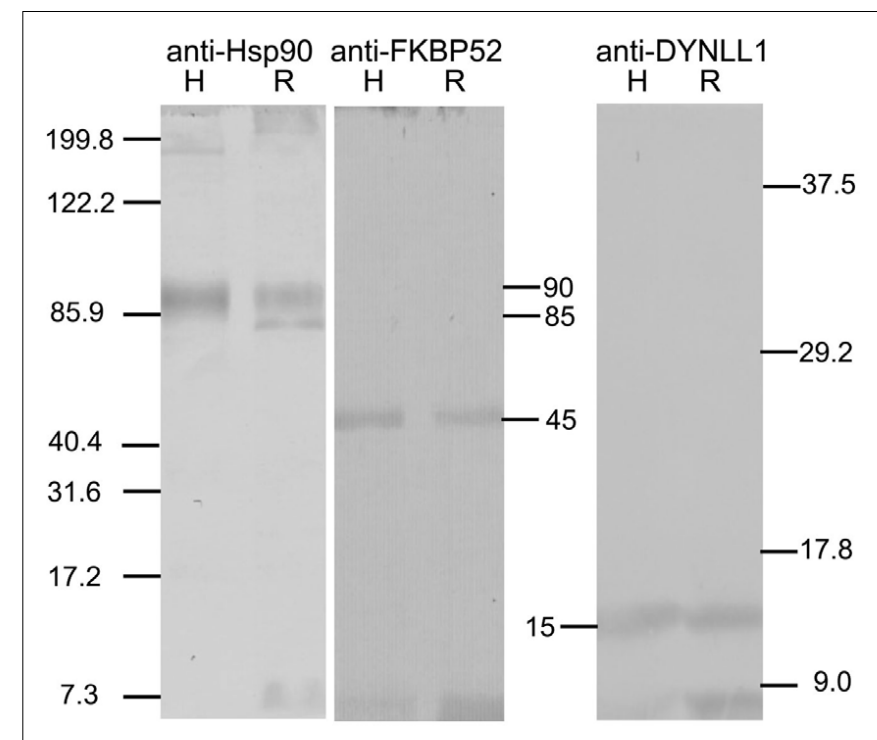

FIGURE 1 | Western blots showing immunoreactivity of control human proteins Hsp90, FKBP52 and DYNLL1 (lanes labeled H), and proteins extracted from Rhodnius SG cells (lanes labeled R) to anti-Hsp90 (left panel), anti-FKBP52 (middle panel), and anti-DYNLL1 (right panel) respectively. The standards are shown on left and right. The relative molecular sizes of the proteins recognized by these antibodies are shown in the middle. Two proteins approximately at 90 and $85 \mathrm{kDa}$ immunoreact with the anti-Hsp90 in SG cell extracts; the 90-kDa protein co-migrates with the 90-kDa control Hsp90. A Rhodnius protein at approximately $45 \mathrm{kDa}$ immunoreacts with anti-FKBP52 and co-migrates with the control FKBP52 protein. A Rhodnius protein at approximately $15 \mathrm{kDa}$ immunoreacts with anti-DYNLL1 and co-migrates with the control DYNLL1 protein.

of an EcR rhythm in various cell types using the 9B9 EcR antibody (Figure 2). Tissues which are known ecdysteroid targets (Vafopoulou et al., 2005), were dissected twice on day 13, in the middle of scotophase ( $7 \mathrm{~h}$ after lights-off) and in the middle of photophase ( $7 \mathrm{~h}$ after lights-on). In scotophase, strong and abundant nuclear EcR fluorescence was observed in all cell types (Figures 2A,C,E,G,I,K) accompanied by moderate (arrows in Figures 2C,E,G) or nil cytoplasmic EcR fluorescence, suggesting genomic effects of ecdysteroids at this time. Both PG and SG cells possess enormous polyploid nuclei carrying a large number of nucleoli (non-fluorescent areas in nuclei; Figures 2C,E respectively); SG cells are binucleate (Figure 2E). The nucleoli were not labeled with the exception of oenocytes (Figure 2K) suggesting that ecdysteroids may also affect nucleolar activities like rRNA synthesis in this cell type.

In contrast, in photophase, widespread distribution of EcR was evident in the cytoplasm of all cell types (Figures 2B,D,F,H,J,L), whereas nuclear EcR fluorescence was significantly diminished, but not entirely absent (e.g., Figures 2B,D,H,L). Cytoplasmic EcR usually appeared either as densely packed fluorescent dots and small rods over most of the cytoplasm (Figures 2F,H) or diffuse and uniform (Figures 2B,D). In several cell types such as the fat body cells (Figure 2H) and oenocytes (Figure 2L), EcR fluorescence exhibited a distinct pattern of accumulation around the nuclei similar to that seen with the distribution of mitochondria in 


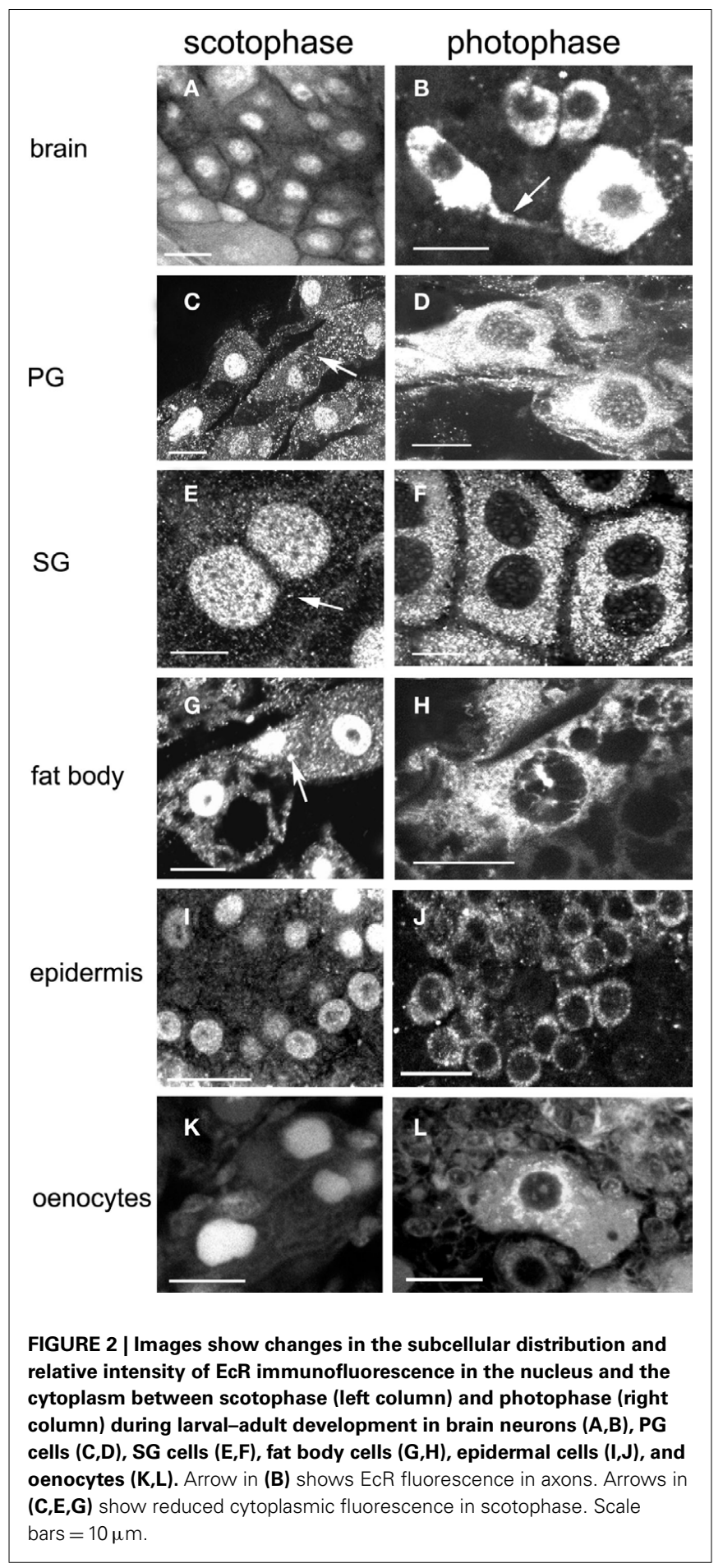

Figure 10. Importantly, EcR fluorescence was also seen in the axons of brain neurons, indicating axonal transport of EcR (Figure 2B, arrow; see Discussion). The cytoplasm of fat body cells was highly vacuolated. These vacuoles represent the areas of fat deposits that were not preserved with paraformaldehyde-glutaraldehyde and were extracted during fixation (Figures 2G,H). Two conclusions are derived from these findings: first, a clear daily rhythm of nuclear EcR fluorescence exists in many cell types of Rhodnius at the beginning of the late period of larval-adult development, confirming our previous findings. Second, this daily rhythm shows daily shuttling of EcR to the nucleus.

\section{DEVELOPMENTAL PROFILES OF ECR FLUORESCENCE IN NUCLEUS AND CYTOPLASM IN DIFFERENT CELL TYPES}

Developmental profiles of EcR distribution in the cytoplasm and nuclei of the cell types described in the previous section were constructed based on cytological evaluation for the presence or absence of EcR fluorescence during the entire larval-adult development (Figure 3). Tissues were dissected throughout this period, beginning at $2 \mathrm{~h}$ after feeding and every 2 days thereafter until day 18; ecdysis occurred around day 21. Dissections were carried out twice a day, once in middle photophase and once in middle scotophase. These time points were selected because EcR fluorescence is confined at these times to either the nucleus or the cytoplasm (see Figure 2). At each time point, 10 animals were sacrificed and whole mounts of six different tissues were scored for the presence of EcR fluorescence in the nuclei and in the cytoplasm. Cytoplasmic EcR fluorescence was scored from the photophase tissues (light bars in Figure 3) and nuclear EcR fluorescence was scored from the scotophase tissues (dark bars in Figure 3). In both cases, fluorescence was scored as either positive (hatched regions of bars) or as negative (unhatched regions of bars). A positive score required at least one cell in each tissue sample to show fluorescence, while a negative score required all cells in each sample to be devoid of fluorescence. Therefore, the bars in Figure 3 do not imply quantitative differences. The bars show the periods of time (days) during development where nuclear and cytoplasmic EcR fluorescence is present in each of the six tissues. It is striking that this time period is tissue-specific (see below). Daily shuttling of EcR between cytoplasm and nucleus can only occur on days when both light and dark bars are hatched, showing that shuttling is constrained to different developmental times in different tissues (see below).

Two general conclusions can be made: first, within a particular cell type, nuclear EcR fluorescence was restricted to a specific developmental period. For example, abundant nuclear EcR fluorescence was apparent almost throughout development in cells of the PGs, epidermis, and fat body. In contrast, nuclear fluorescence was present during short periods of time in brain neurons, SG cells, and oenocytes. These profiles are in general agreement with previous findings and their importance to development has been discussed elsewhere (Vafopoulou et al., 2005). Second, the profiles of cytoplasmic EcR fluorescence corresponding to a particular cell type are rarely completely coincidental within a day with those of nuclear EcR fluorescence. This is the case for all cell types except PG cells in which both nuclear and cytoplasmic EcR fluorescence were present throughout development. In three cell types, brain neurons, SG cells, and oenocytes the presence of EcR fluorescence in the nuclei was brief whereas the appearance of cytoplasmic EcR fluorescence was significantly longer; in the case of brain neurons in particular, presence of cytoplasmic EcR fluorescence spanned almost throughout development. Curiously, in fat body and epidermal cells nuclear EcR fluorescence appeared almost immediately after feeding, whereas cytoplasmic EcR fluorescence appeared only 6 days later. We conclude, first that 


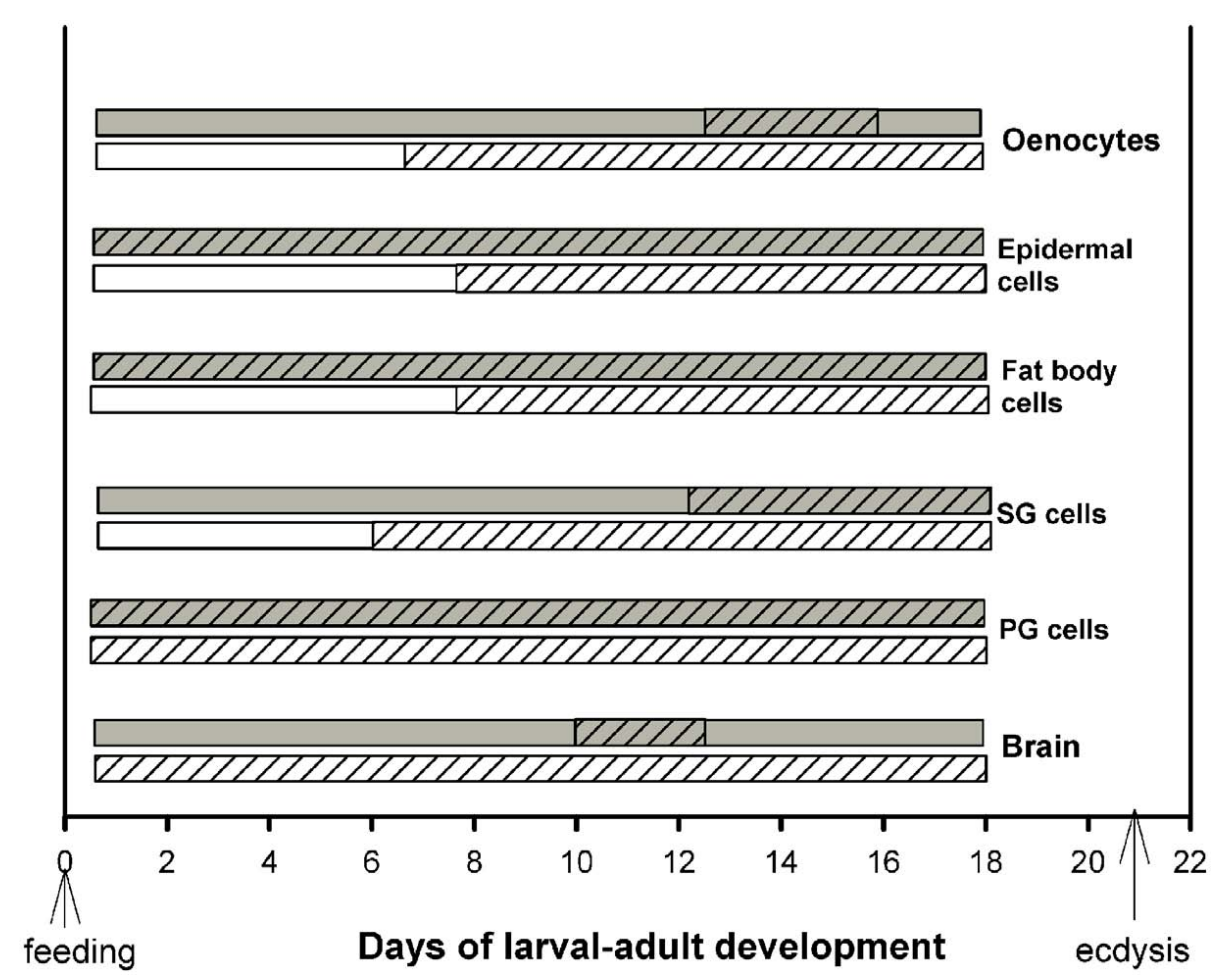

FIGURE 3 | Schematic diagram of the periods during development (in days after feeding) during which cytoplasmic (light bars; day) and nuclear (dark bars; night) EcR fluorescence was found in six different tissues. Hatched areas of each bar show the length of time (in days) during which EcR fluorescence is seen in the cytoplasm during the day and in the nucleus during the night of each tissue. Note that EcR is present for different lengths of developmental time in different tissues. Cytoplasmic-nuclear shuttling of EcR is necessarily confined to the developmental times when both the light and dark bars are hatched, i.e., EcR is present in both compartments during the same $24 \mathrm{~h}$ period. For details, see text. presence of EcR in the cytoplasm is a widespread phenomenon in Rhodnius cells. Second, during larval-adult development nuclear and cytoplasmic EcR are seen in the same $24 \mathrm{~h}$ period only at certain cell-specific periods during the course of development. This observation indicates that daily shuttling between cytoplasm and nucleus (see Cycling of EcR in Rhodnius cells) is also confined to certain specific time periods during development and that these periods vary between cell types. These periods vary in duration and their position on the developmental time scale depending on the cell type. In general, during development, EcR fluorescence is found more frequently in the cytoplasm than in the nucleus. The finding of cytoplasmic EcR throughout extended periods of development raises interesting questions as to the significance of EcR in the cytoplasm of Rhodnius cells. The spatial relationship of EcR with cytoplasmic organelles suggests functions for cytoplasmic EcR.

\section{CO-LOCALIZATION OF EcR WITH MTs}

The spatial relationship of cytoplasmic EcR fluorescence with MTs was determined in double label experiments using the EcR antibody and an antibody against tyrosilated $\alpha$-tubulin (Figures 4 and 5). Tissues were obtained from scotophase animals at day 13. Several types of cells were pre-treated with taxol which stabilizes MTs against disassembly and promotes excess assembly. Taxol caused a marked reduction in the relative intensity of EcR fluorescence in the nucleus (compare left column of Figure 2 with left columns in Figures 4 and 5). Therefore, it appears that MT stabilization inhibits the accumulation of EcR within the nucleus.

Ecdysteroid receptor fluorescence is shown in the left column (green), tubulin fluorescence is shown in the right column (red). Co-localization is shown as yellow/orange in the merged column; the cytoplasmic areas enclosed in small white rectangles in the merged column are shown enlarged in the enlarged column from the left. Extensive networks of MTs were revealed with anti-tubulin label in PG cells (Figures 4A-C), brain neurons (Figures 4D-I), SG cells (Figures 4J-L), fat body cells (Figures $4 \mathrm{M}-\mathrm{O}$ ), oenocytes (Figures 5A-F), and epidermal cells (Figures 5D-F). In epidermal cells (Figure 5E) and oenocytes (Figures 5C,F) in particular, MTs were arranged radially from well defined centrosomes. Strong cytoplasmic EcR fluorescence is seen in all cell types, which exhibited a closely similar distribution to that of MTs and extensive co-localization with MTs (merged columns in Figures 4 and 5); this is particularly evident in oenocytes (Figures 5A,D) and epidermal cells (Figure 5D), where specks of EcR fluorescence follow closely the radial pattern of MTs within the cell bodies (Figures 5B1,E1, arrows). The enlarged images (enlarged columns) show clearly that EcR fluorescence forms punctate spots that are aligned along MTs. Therefore, an intimate spatial relationship exists between EcR fluorescence and tubulin fluorescence suggesting a potential association between 


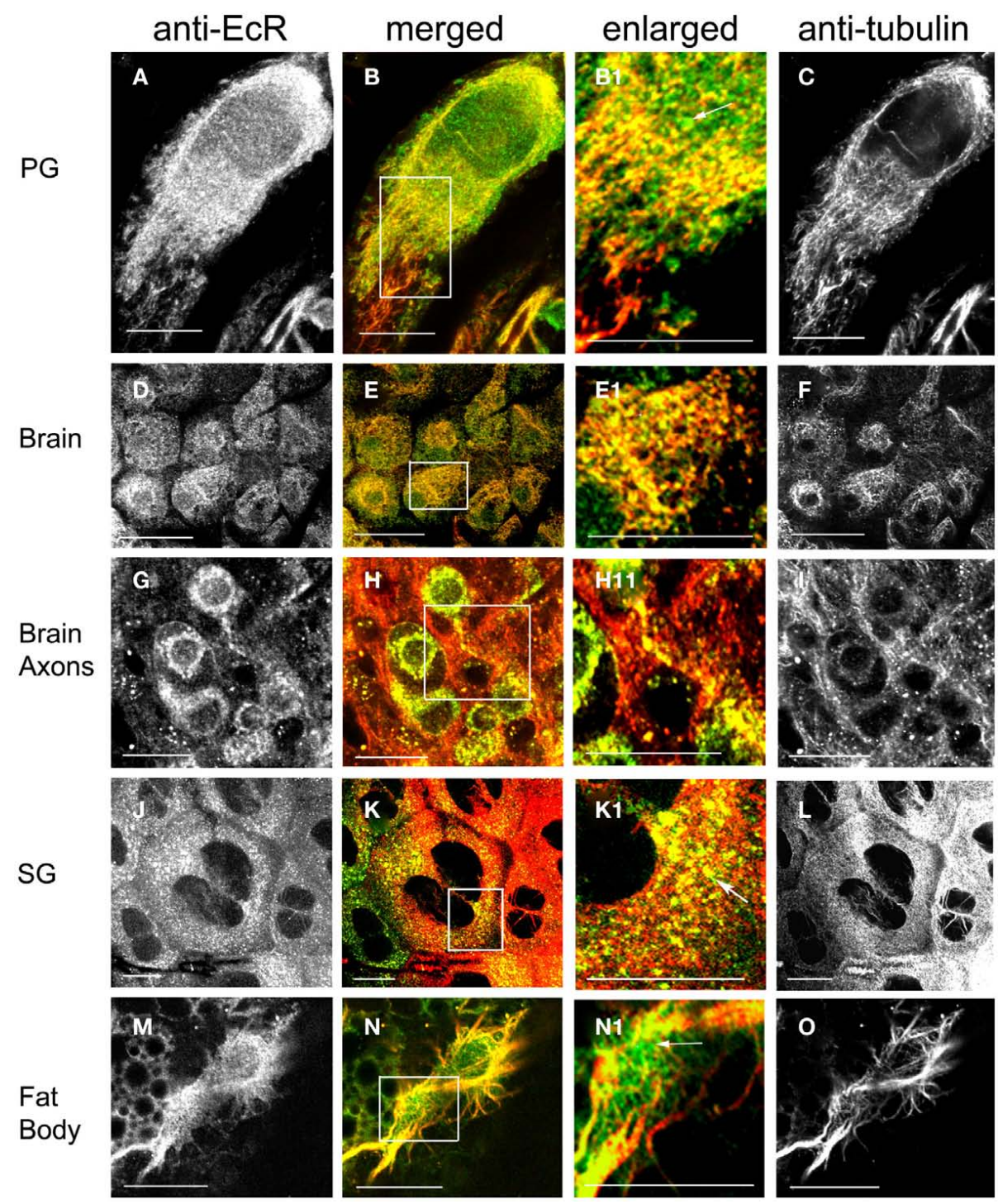

FIGURE 4 | Cells double-labeled with anti-EcR (left column, green) and anti-tubulin (right column, red). Merged images of left and right are shown in the merged column. Co-localization is shown as yellow/orange. Cells were pre-incubated with the MT-stabilizing agent taxol. PG cells (A-C), brain neurons (D-I), SG cells (J-L), fat body cells $\mathbf{( M - O )}$. Areas enclosed in the white lined boxes in $(\mathbf{B}, \mathbf{E}, \mathbf{H}, \mathbf{K}, \mathbf{N})$ are shown in the enlarged column as $(\mathbf{B 1}, \mathbf{E 1}, \mathbf{H 1} \mathbf{K} \mathbf{1}, \mathbf{N 1})$ respectively; these images show details of co-localization of EcR fluorescence with tubulin fluorescence on MTs. Note the alignment of EcR fluorescence with MTs in these images; this is particularly evident in (H1) that shows an axon. Arrows in $\mathbf{( B 1 , K 1 , N 1 ) ~ s h o w ~ t h a t ~ n o t ~ a l l ~ E c R ~ f l u o r e s c e n c e ~ c o - l o c a l i z e s ~}$ with MTs. Scale bars $=10 \mu \mathrm{m}$.
EcR and MTs. This co-localization between MTs and EcR was also observed in axons of brain neurons shown in Figure 4H1, suggesting that EcR may be moved along axons (above) by MTs. However, it should be noted that not all cytoplasmic EcR fluorescence co-localized with MTs; this is evident in Figures 4B1,K1,N1 and 5B1,E1 (arrows).

Pre-treatment of scotophase cells with colchicine, a MT disrupting agent, disrupted the MT network, and anti-tubulin fluorescence became re-distributed into discrete cytoplasmic pockets in the form of bright dots (Figure 6, right column). The colocalization of tubulin and EcR fluorescence persisted in these pockets (Figure 6, merged column). Critically, the nuclear EcR fluorescence was greatly diminished (Figure 6, left column). This pattern of distribution of EcR fluorescence resembles that of the photophase (see Figure 2) rather than the scotophase. Therefore, disruption of MTs causes a loss of nuclear EcR fluorescence (like that with stabilization of MTs by taxol) and a re-distribution of cytoplasmic EcR fluorescence into the photophase configuration rather than that of the scotophase. We conclude that some EcR co-localizes with MTs in various cell types of Rhodnius, suggesting that EcR may be transported within the cytoplasm using the MT network. 

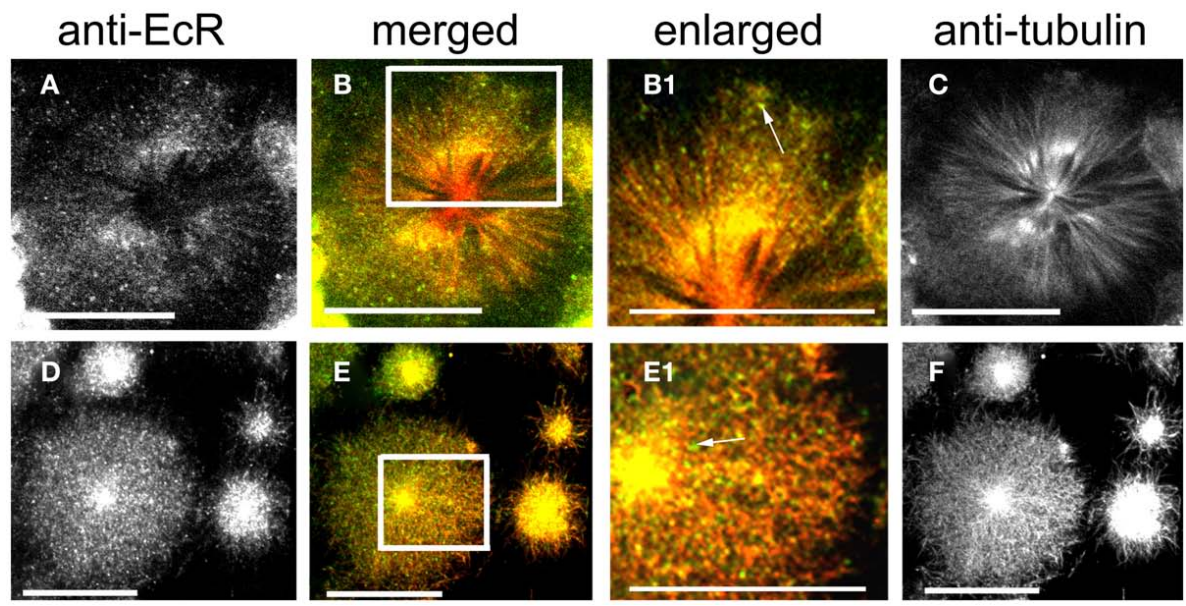

FIGURE 5 | Cells double-labeled with anti-EcR (left column, green) and anti-tubulin (right column, red). The merged column shows merged images of left and right. Co-localization is shown as yellow/orange. Cells were pre-incubated with the MT-stabilizing agent taxol. Large cells are oenocytes and small cells are epidermal cells. Both rows show different aspects of oenocytes. (C,F) Show the radial arrangement of MTs from a well defined centrosome. Areas enclosed in the white lined boxes in $\mathbf{( B , E )}$ are shown in the enlarged column as (B1,E1) respectively. Note the co-localization of EcR fluorescence with MTs (yellow specks) in (B1,E1). Some EcR fluorescence does not associate with MTs [green specks, arrows in (B1,E1)]. Scale bars $=10 \mu \mathrm{m}$.

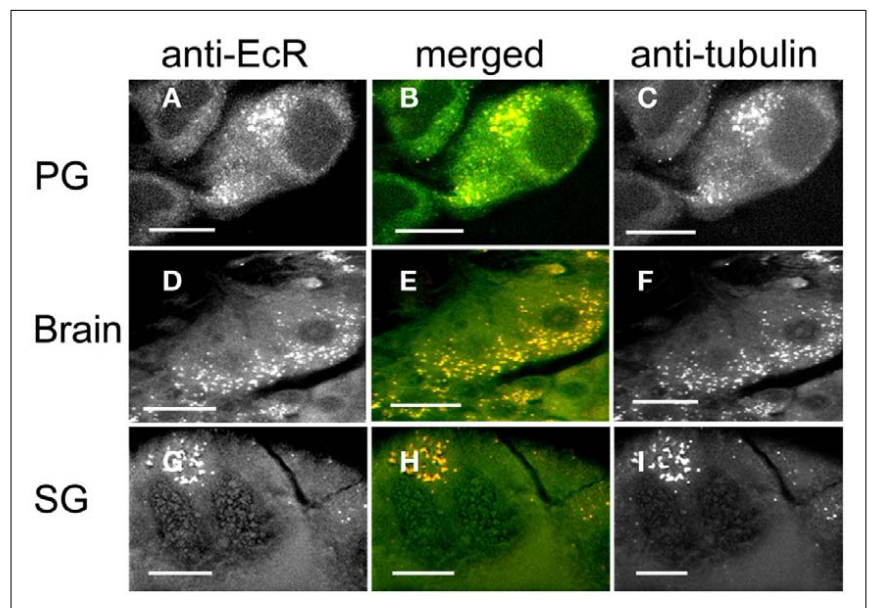

FIGURE 6 | PG cells (A-C), brain neurons (D-F), and SG cells (G-I) pre-treated with colchicine to disrupt MTs and double-labeled with anti-EcR (left column, green) and anti-tubulin (right column, red). Co-localization is shown as yellow/orange in the merged images of left and right in the middle column. Note the disruption of MTs in (C,F,I), which appear as fluorescent specks and re-distribution of tubulin fluorescence. Co-localization is retained after colchicine treatment $\mathbf{( B , E , H ) . ~ S c a l e ~}$ bars $=10 \mu \mathrm{m}$

\section{CO-LOCALIZATION OF EcR WITH THE MOLECULAR CHAPERONE Hsp90}

Co-localization of EcR with the molecular chaperone Hsp90 was examined in SG cells. Rhodnius SGs consist of a single lobe which is composed of an epithelial monolayer of flat binucleate cells (about 6-8 $\mu \mathrm{m}$ thick) surrounding the lumen that contains the salivary secretions. These cells are very large (about $40 \mu \mathrm{m}$ in diameter) which makes them ideal for cytological analysis of their cytoplasmic constituents. The cellular distribution of Hsp90 (Figure 7 right, red) and its co-localization with EcR (Figure 7 left, green) were both revealed in double label experiments with antibodies against both proteins (Figures 7A-L). Hsp90 was seen in abundance forming a punctate pattern in the cytoplasm of cells in photophase (Figures 7I,L,M), where much, but not all, Hsp90 colocalized with EcR (EcR seen in Figures 7G,J). Co-localization is seen in particular in the merged images in Figures $7 \mathbf{H}, \mathbf{K}$ and the enlarged images in Figures $7 \mathbf{H 1}, \mathbf{K} 1$; the abundance of Hsp90 in these images agrees with its ubiquitous presence in animal cells. Likewise, not all of the green specks of EcR fluorescence co-localized with the red specs of Hsp90 fluorescence (Figure 7H1), showing that only a portion of cytoplasmic EcR is associated with Hsp90. This close spatial relationship of EcR with Hsp90 suggests that an EcR-Hsp90 receptor-chaperone complex may be formed, as is common with other steroid receptors (see Discussion).

In nuclei from the scotophase, Hsp90 was found as a diffuse stain in many cells of the same sample (Figure 7F), (but not all, Figure 7C), where it co-localizes with EcR fluorescence (yellow/green nuclei in Figure 7E). When co-localization of Hsp90 and EcR was seen in nuclei, Hsp90 presence in the cytoplasm was considerably reduced (Figure 7E1; compare with Figure 7B1) and no co-localization was seen with EcR, suggesting that most EcR had been transported to the nucleus during the scotophase (compare Figures 7A,D with Figure 7G, which shows nuclei in photophase). Images Figures 7C,F suggest the existence of two different states of Hsp90 in nuclei, one potentially associated with EcR and the other potentially dissociated from it.

In the cytoplasm, pre-treatment with taxol caused a dramatic re-organization of both EcR dots (Figure 7J) and Hsp90 dots (Figure 7L); both formed elongated rods arranged in lines that presumably correspond to MTs. Even after taxol treatment, the cytoplasmic co-localization of Hsp90 with EcR was maintained (Figures 7K,K1). Double labels with anti-Hsp90 and antitubulin revealed a co-localization of Hsp90 (Figure 7M) with MTs 

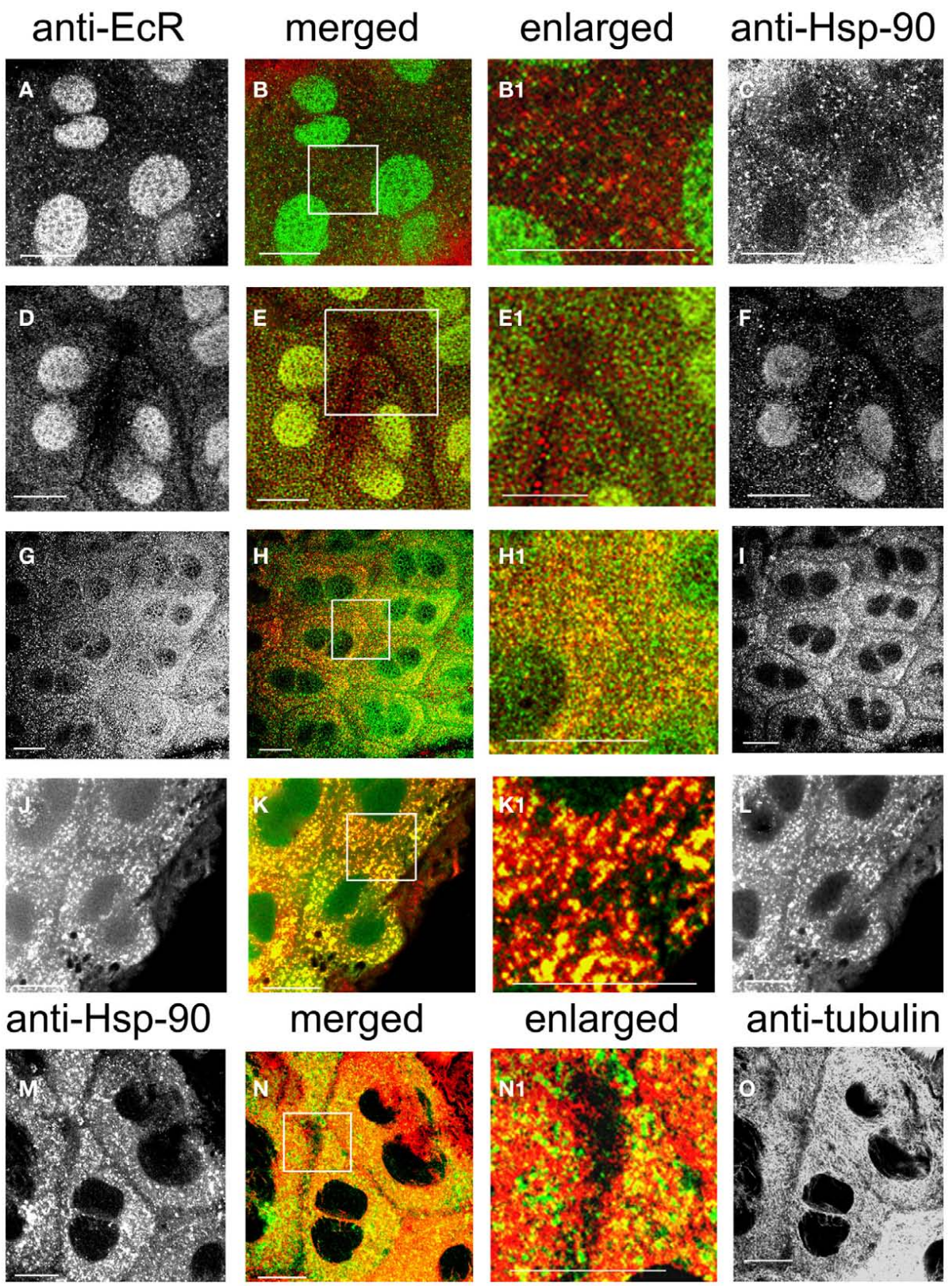

FIGURE 7 | SG cells double-labeled with anti-EcR and anti-HsP90 (A-L). Anti-EcR is shown on the left column (green) and anti-Hsp is shown on the right column (red). (M-O) Shows double labels with anti-Hsp90 (left, green) and anti-tubulin (right, red). The merged column shows the merged images of left and right. Co-localization is shown as yellow/orange. Areas enclosed in the white lined boxes in $(\mathbf{B}, \mathbf{E}, \mathbf{H}, \mathbf{K}, \mathbf{N})$ are shown in the enlarged columns as in (B1,E1, H1, K1,N1) respectively; these images show details of co-localization of EcR fluorescence with Hsp90 fluorescence (B1,E1,H1,K1) and details of co-localization of Hsp90 fluorescence with anti-tubulin (N1). (A-F) Show SG cells in scotophase and (G-I) shows cells in photophase. Note absence of co-localization in the cytoplasm in scotophase labundance of red and green specks in $(\mathbf{B 1}, \mathbf{E 1})$ ] and widespread co-localization in photophase [abundance of yellow/orange specks in (H1)]. (J-L) Shows cells pre-treated with taxol. Note the arrangement of co-localized EcR and Hsp90 fluorescence in yellow lines (K1). In (N1), note co-localization of Hsp90 fluorescence with tubulin fluorescence (yellow specks along MTs). Scale bars $=10 \mu \mathrm{m}$.
(Figure 7O) as seen in Figure 7N; this co-localization was in the form of fine lines along the tracks of MTs as seen in the enlarged image in Figure 7N1. Therefore, the putative EcR-Hsp90 complex is co-localized with the MT network in the cytoplasm of Rhodnius SG cells.

\section{CO-LOCALIZATION OF ECR WITH THE IMMUNOPHILIN FKBP52}

The cellular distribution of immunophilin FKBP52 (Figures 8C,F,I, red) and its spatial relationship with EcR (Figures 8A,D,G, green) was revealed with double labels using antibodies against both proteins (Figures 8A-I). FKBP52 fluorescence appears punctate 
anti-EcR
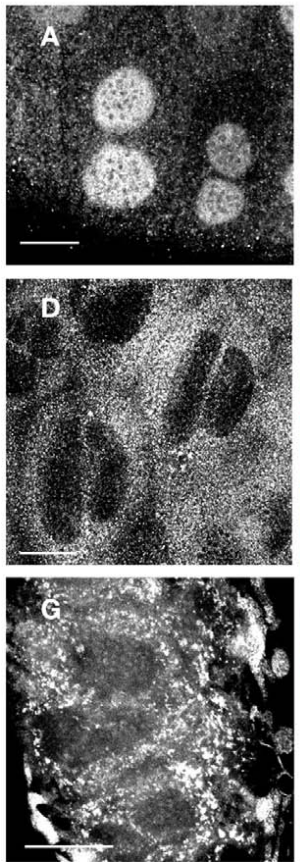

anti-FKBP52

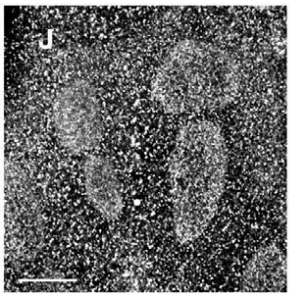

merged
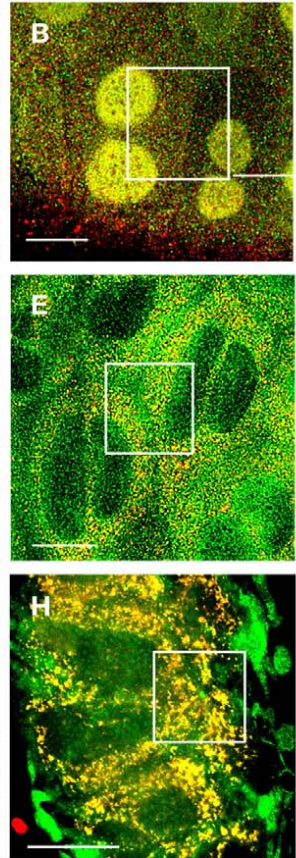

merged

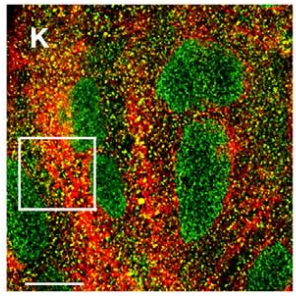

enlarged
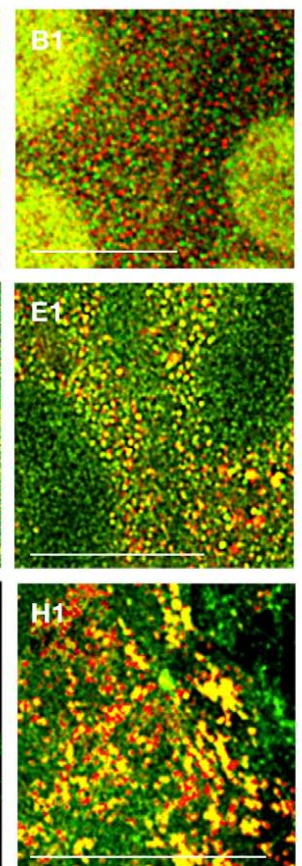

enlarged

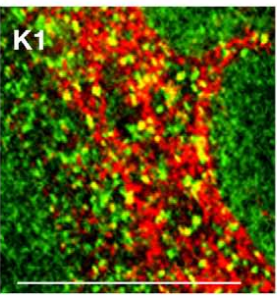

\section{anti-FKBP52}
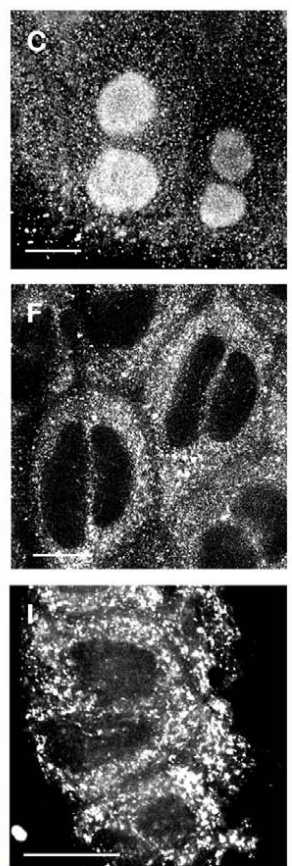

anti-tubulin

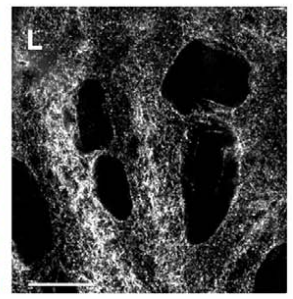

FIGURE 8 | (A-I) Show SG cells double-labeled with anti-EcR (left column, green) and anti-FKBP52 (right column, red). (J-L) Show SG cells double-labeled with anti-FKBP52 [(J), green] and anti-tubulin [(L), red]. The merged column shows merged images of left and right. Co-localization is shown as yellow/orange. Areas enclosed in the white lined boxes in $\mathbf{( B , E , H , K )}$ are shown enlarged in $(\mathbf{B 1}, \mathbf{E 1}, \mathbf{H 1}, \mathbf{K} \mathbf{1})$ respectively; these images show details of co-localization of EcR fluorescence with FKBP52 fluorescence (B1,E1,H1) and details of co-localization of FKBP52 fluorescence with anti-tubulin (K1)
(A-C) Show scotophase and (D-F) show photophase. Note the absence of cytoplasmic co-localization of EcR fluorescence and FKBP52 fluorescence in scotophase and its presence in nuclei [red and green specks in (B1)]. In contrast, note extensive co-localization in the cytoplasm in photophase and its absence from nuclei [yellow specks in (E1)]. (G-I) Show cells pre-treated with taxol. Note the alignment of yellow dots and rods in lines in (H1). In (K1), note co-localization of FKBP52 fluorescence with anti-tubulin (yellow specks along MTs). Scale bars $=10 \mu \mathrm{m}$. and broadly distributed throughout the cytoplasm in photophase (Figure 8F) and is partially co-localized with the green EcR dots (Figure 8E). The enlarged image in Figure 8E1 clearly shows yellow dots, indicating a co-localization of EcR and FKBP52 in the cytoplasm of SG cells. In the nucleus, co-localization is seen in the scotophase, where fluorescence of FKBP52 (Figure 8C) colocalizes with that of EcR (Figure 8A) as shown in Figures 8B,B1, where nuclear stain appears as diffuse yellow/green. In the scotophase, little, or no EcR fluorescence remains in the cytoplasm (see Figure 8B1), and the remaining FKBP52 fluorescence appears as red dots (free FKBP52; compare Figure 8E1 with Figure 8B). Two conclusions can be drawn from these findings: first, EcR may form a complex with the co-chaperone FKBP52 in photophase and second, this complex seems to translocate to the nucleus in scotophase. When photophase SG cells were pre-treated with taxol, the previously scattered dots of EcR and FKBP52 seen in the cytoplasm of untreated cells (see Figures 8E,E1), became re-organized to elongated rods (Figure 8G for EcR fluorescence and Figure 8I for FKBP52 fluorescence) that aligned in tandem in the direction of MTs as seen in the merged images in Figures $\mathbf{8 H}, \mathbf{H 1}$. This alignment of EcR-FKBP52 into rows suggests they may attach to MTs. This inference is supported by double labels of scotophase SGs with anti-FKBP52 (Figure 8J) and anti-tubulin (Figure 8L), in which FKBP52 fluorescence co-localizes with MTs as shown in the merged image in Figure $\mathbf{8 K}$ and the enlarged image in Figure 8K1. These observations suggest that FKBP52 is probably attached to MTs. We conclude that the inferred EcR-FKBP52 complex co-localizes with MTs. 


\section{CO-LOCALIZATION OF ECR WITH LIGHT CHAIN 1 OF DYNEIN}

The co-localization of EcR with the light chain 1 of dynein, DYNLL1, was revealed in photophase SG cells with triple labels using antibodies against EcR (Figure 9A; green), tubulin (Figure 9B; red) and dynein (Figure 9C; blue). Staining of cytoplasmic dynein was punctate throughout the cytoplasm (Figure 9B) and co-localized with both EcR and tubulin (Figure 9D). Co-localization of all three appears as white dots throughout the cytoplasm, which appear to follow the tracks of the MT as seen in the enlarged area in Figure 9D1. It is therefore probable that that EcR co-localizes with both DYNLL1 and tubulin.

\section{CO-LOCALIZATION OF ECR WITH MITOCHONDRIA}

The spatial relationship of EcR and mitochondria was examined in brain neurons (Figures 10A-C), PGs (Figures 10D-F), SGs (Figures 10G-I), epidermal cells (Figures 10J-L), and fat body cells (Figures 10M-O) in double label experiments using anti-EcR (left column, green) and MitoTracker Deep Red 633 (right column, red); co-localization is shown as yellow/orange in the merged column. All dissections were carried out in photophase. Mitochondria were widely distributed in the cytoplasm of all cells forming fluorescent webs with a relatively high density in the perinuclear region; this is particularly evident in PG cells (Figure 10F), epidermal cells (Figure 10L), and fat body cells (Figure 10O). The distribution of mitochondria in fixed cells closely reflects that in live cells, where the mitochondria tend to aggregate in the perinuclear area of cells, showing that fixation preserved organelle structure satisfactorily (not shown). In all cell types there was considerable co-localization of EcR fluorescence with that of MitoTracker (arrows in the merged column). However, not all EcR fluorescence co-localized with mitochondrial fluorescence as it is seen as a widespread green label in Figures 10B,E,H,N. Likewise, not all mitochondrial fluorescence co-localized with EcR

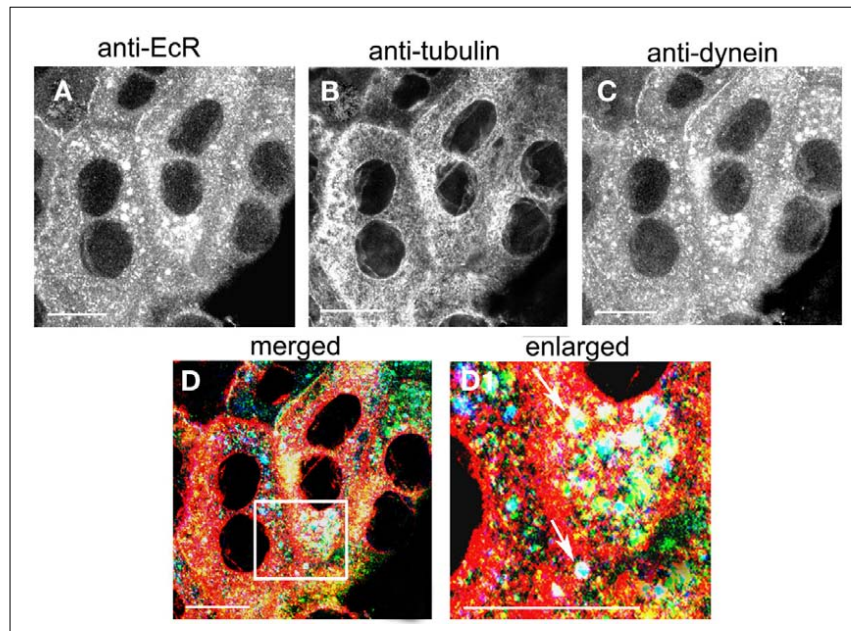

FIGURE 9 | Triple labeling of SG cells with anti-EcR [(A), green], anti-tubulin [(B), red] and anti-dynein [(C), blue]. Co-localization is shown in (D,D1) as white. Area enclosed in the white lined boxes in (D) is shown enlarged in (D1) to show details of co-localization. Note co-localization of EcR, tubulin, and dynein fluorescence in (D1) as white dots (arrows). Scale bars $=10 \mu \mathrm{m}$. fluorescence (seen as red specks in the middle column). The degree of co-localization depended on the cell type. Brain neurons (Figures 10A-C shows a large neurosecretory cell and smaller neurons), PG cells (Figures 10D-F), SG cells (Figures 10G-I), and fat body cells (Figures 10M-O) showed moderate co-localization whereas, epidermal cells (Figures 10J-L) showed extensive colocalization. Therefore, a considerable amount of, but not all, cytoplasmic EcR fluorescence, co-localizes with mitochondria in Rhodnius cells, depending on the cell type.

\section{ABSENCE OF CO-LOCALIZATION OF ECR WITH ACIDIC ORGANELLES}

The spatial relationship of EcR with cytoplasmic acidic organelles, such as lysosomes, was examined in double label experiments (Figure 11) using anti-EcR (left column; green) and LysoTracker (right column; red); co-localization is shown in the central column as orange/yellow. Lysosomes have been implicated in EcR elimination in some animals (see Discussion). Animals were selected at the same developmental days as above. None of the cell types tested showed any significant co-localization of EcR fluorescence with LysoTracker fluorescence as in the examples shown Figures 11A-C for brain neurons and Figures 11D-F for SG cells. We conclude that EcR in Rhodnius does not appear to associate with acidic organelles.

\section{DISCUSSION \\ NUCLEOCYTOPLASMIC SHUTTLING OF ECR AND THE ROLE OF MICROTUBULES}

The temporal and spatial profiles of EcR reported here during larval-adult development, revealed that shuttling between the nucleus and cytoplasm was restricted to specific developmental periods in most cell types. In many cases, the presence of EcR in the cytoplasm exceeded the period of shuttling. This finding emphasizes that not all of the cytoplasmic EcR is necessarily involved in shuttling. This conclusion raises the prospect that EcR may also be involved in specific cytoplasmic events, i.e., non-genomic actions. Further investigation of the function(s) of cytoplasmic EcR is clearly required. In Rhodnius, daily shuttling of EcR to the nucleus implies EcR transport mechanisms. Extensive work with mammalian nuclear receptors has established that receptors employ various methods for nuclear import, including conventional nuclear localization signal machinery or use of cytoskeletal tracks (review by Echeverria and Picard, 2010). Conventional machinery involving nuclear signaling has been until now assumed the only mechanism of intracellular EcR movement (reviews by Henrich, 2009; Nakagawa and Henrich, 2009).

We studied details of nucleocytoplasmic trafficking of EcR in Rhodnius cells. We found that in a variety of cells types, substantial amounts of EcR co-localized with the MT network when MTs were stabilized with taxol. In epidermal cells and oenocytes in particular, where well defined centrosomes were present, specks of EcR fluorescence were detected attached to radiating lines of MTs (see Figure 5). This relationship of EcR with the cytoskeleton in insects has only been reported before in Rhodnius PG cells (Vafopoulou, 2009). No such association has been reported for any other insect. In contrast, this association is quite common in mammals where shuttling of nuclear receptors in and out of the nucleus has been the focus of in depth study. Association of steroid receptors with 


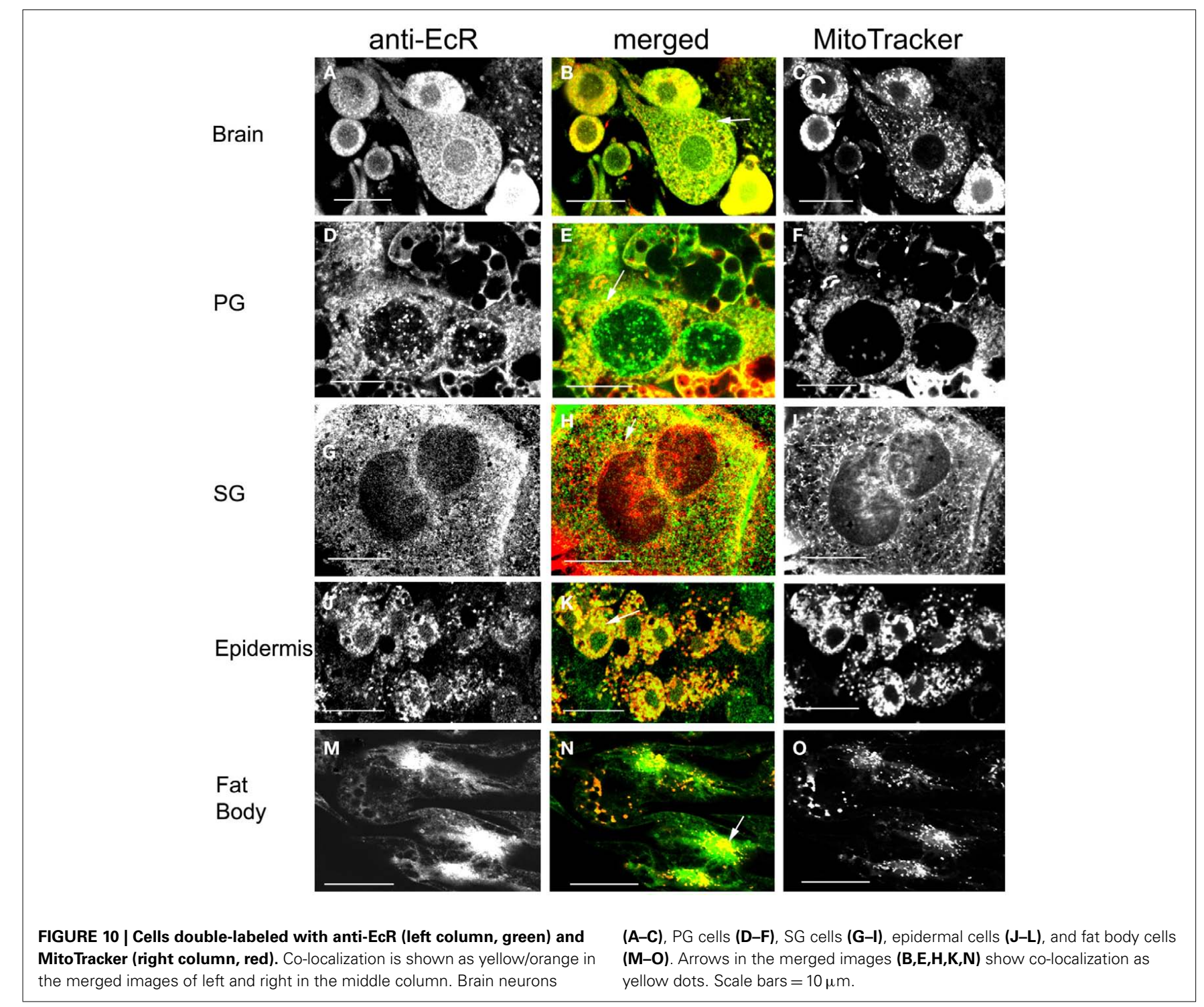

MTs has been reported, both for the ligand-free receptor (e.g., GR, Akner et al., 1991; reviews by Akner et al., 1995 and by Dvorák et al., 2004) and for the ligand-bound receptor (e.g., estrogen receptor $\alpha$, Azuma et al., 2004; Manavathi et al., 2006; progesterone receptor, Shimazu, 2003; GR, Galigniana et al., 1998, 2001; Dvorák et al., 2005, 2007; Harrell et al., 2004; vitamin D receptor, Barsony et al., 1997). These precedents raise the prospect that MT may play a critical role in EcR shuttling in Rhodnius and potentially in insects in general.

Our present and previous data (Vafopoulou, 2009) showed that treatment of scotophase cells with the MT-stabilizing agent taxol resulted in a considerable reduction in nuclear EcR with the concomitant increase in cytoplasmic EcR. This finding suggests that MT stabilization inhibits receptor accumulation in the nucleus and further supports the notion that MTs may serve as movement machinery for EcR. Possibly, stabilization of MTs prevents movement of EcR to the nucleus by tethering it to MTs. Evidence in support of this idea was provided by Manavathi et al. (2006) who found that taxol inhibited ERa transcriptional activity in human breast cancer cells and taxol increased tubulin association with the mineralocorticoid receptor (MR)-Hsp90 heterocomplex (Galigniana et al., 2010). We also observed that disruption of MTs in cells using colchicine (Vafopoulou, 2009 and present evidence) resulted in depletion of nuclear EcR, suggesting that destruction of cytoskeletal tracks also inhibited transport of EcR from cytoplasm to nucleus. A comparable inhibition of nucleocytoplasmic shuttling of GR and aryl hydrocarbon receptor by agents that disrupt MTs was reported by Dvorák et al. (2007). In colchicinetreated cells of Rhodnius, the association of EcR with tubulin was maintained but became fragmented into scattered dots in pockets of cytoplasm with the normal organized network of MTs being lost. This finding again supports the idea that nucleocytoplasmic shuttling of EcR requires an intact cytoskeleton. The directional movement through the cytoplasm to the nucleus using MTs implied by the above argument clearly indicates genomic action of EcR. 


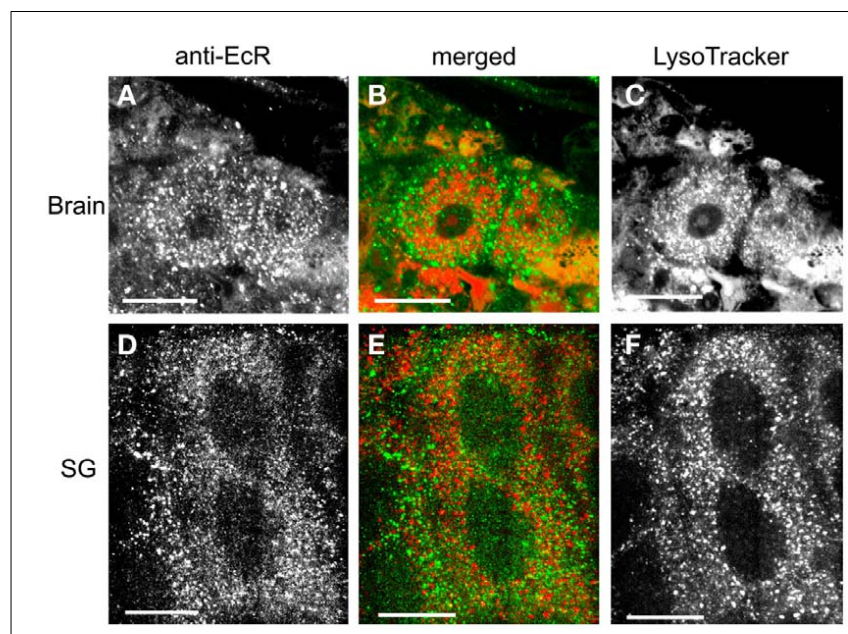

FIGURE 11 | Cells double-labeled with anti-EcR (left column, green) and LysoTracker (right column, red). The middle column shows merged images of left and right. Brain neurons (A-C) and SG cells (D-F). Note the low level of co-localization in the merged images in the middle column. EcR fluorescence and fluorescence from LysoTracker labeling are shown as distinct red and green dots in the cytoplasm. Scale bars $=10 \mu \mathrm{m}$.

\section{RELATIONSHIPS OF EcR WITH THE CHAPERONE Hsp90 AND THE FKBP52 IMMUNOPHILIN}

Proteins require molecular chaperones for protection from proteolysis, normal folding, and maturation. Generally, steroid receptors form large multiprotein heterocomplexes with the ubiquitous and essential chaperone proteins, the heat shock proteins 90 and 70 (Hsp90 and Hsp70) and many co-chaperones including the immunophilins FKBP51 and FKBP52 that bind to an acceptor site on Hsp90 via a tetratricopeptide repeat (TPR; reviews by Nollen and Morimoto, 2002; Pratt and Toft, 2003; Pratt et al., 2004a,b). Immunophilins are intracellular receptors for immunosuppressant drugs such as FK506, rapamycin, and cyclosporine A and have been implicated as modulators of steroid receptor function (Ratajczak et al., 2003).

We used antibodies against human Hsp90 and FKBP52 to localize intracellularly related proteins within Rhodnius SG cells. We used these cells as model cells for Rhodnius because of their large size, flat morphology, and expansive cytoplasmic area. Hsp90 is a ubiquitous and highly conserved protein crucial to cellular signaling involved in numerous cellular regulations including steroid hormone signaling, the regulation of folding, activity, and stability of a wide range of client proteins such as steroid receptors (reviews by Pratt et al., 2004a,b; Picard, 2006; Echeverria and Picard, 2010). The FK506-binding protein 52, FKBP52, is part of the mature steroid receptor heterocomplex and it plays a crucial role in the intracellular trafficking of receptors (reviews above). On Western blots of SG protein extracts, the antibody against human Hsp90 recognized a peptide around $90 \mathrm{kDa}$ and the antibody against human FKBP52 recognized a peptide of molecular size about $45 \mathrm{kDa}$ showing that Rhodnius contains immunoreactive peptides of similar molecular size to their human equivalents. Double immunolabels showed extensive and intimate co-localization of EcR with both the ubiquitous chaperone
Hsp90 and the co-chaperone FKBP52. These close spatial relationships provide the first evidence that EcR may associate with both chaperone proteins, suggesting that these proteins may form heterocomplexes with EcR in Rhodnius as they do with mammalian steroid receptors. Information is scarce about the requirement of chaperones or even their presence, for EcR function, with a few exceptions. For example, purified EcR/USP heterodimer from Drosophila acquires DNA binding activity only with the addition of chaperones such as Hsp90 and FKBP52 (Arbeitman and Hogness, 2000). Also, FKBP52 was immunoprecipitated along with EcR in the PGs of M. sexta, raising the possibility of a functional role of this immunophilin in this insect (Song et al., 1997).

Importantly, both Hsp90 and FKBP52 co-localize with MTs in Rhodnius SG cells. Association of FKBP52 with MTs and tubulin in particular has also been shown in mammalian cells (PerrotApplanat et al., 1995; Chambraud et al., 2007). Since EcR colocalizes with both Hsp90 and FKBP52 and all three proteins also co-localize with MTs, it is reasonable to suggest that the EcR association with MTs occurs in the form of a EcR-Hsp90-FKBP52 heterocomplex as does its mammalian counterparts (see below).

Interestingly, the intimate association of EcR with either chaperone is maintained in both the photophase and scotophase. In the photophase, associations of EcR with either Hsp90 or FKBP52 appear as scattered fluorescent dots in the cytoplasm with no fluorescence present in the nucleus. On the other hand, in scotophase, both Hsp90 and FKBP52 fluorescence co-localize with EcR fluorescence in the nucleus. At the same time, the cytoplasm becomes largely devoid of fluorescent dots from co-localization of EcR with either chaperone, suggesting that the bulk of EcR has become transported to the nucleus as a complex with Hsp90 and FKBP52. In scotophase, we detected EcR-positive nuclei both with and without Hsp90 in the same tissue sample, which is consistent with the expectation that Hsp90 dissociates from the receptor within the nucleus following transport. Dissociation from FKBP52 may also occur inside the nucleus. A widely accepted model is that the receptor-Hsp90 based heterocomplex dissociates in the cytoplasm following receptor transformation by ligand binding. However, recent evidence showed that the MR-Hsp90immunophilin complex accumulates in the nucleus in a hormonedependent manner and dissociates within the nucleus (Galigniana et al., 2010), i.e., receptor transformation occurs inside the nucleus. Even in the absence of ligand, GR-Hsp90-immunophilin complex can translocate into the nucleus as an intact complex (Echeverría et al., 2009). Our results are consistent with the view that dissociation of EcR from the chaperones Hsp90 and FKBP52 occurs within the nucleus, indicating that transformation of the receptor occurs inside the nucleus. Considering the fact that daily EcR shuttling occurs in synchrony with daily ecdysteroid peaks in the hemolymph (Vafopoulou and Steel, 2006; review by Steel and Vafopoulou, 2006), we envisage that EcR is transformed rhythmically every day inside the nucleus in response to daily pulses of the ligand.

\section{RELATIONSHIP OF ECR WITH CYTOPLASMIC DYNEIN AND ROLE OF THE COMPLEX IN ECR TRAFFICKING}

The cytoskeleton is considered a vital biological network for integration of signal transduction pathways. Bi-directional transport 
of cargo is achieved by motor-driven structures like MTs (review by Welte, 2004). MTs have plus and minus ends. They are organized in such a way that their minus ends are clustered around the centrosome, which is located near the nucleus and their plus ends are near the periphery. This arrangement is suitable for transport of cargo from the cytoplasm to the nucleus and back. Cytoplasmic dynein is a multisubunit high molecular weight ATPase that transports cargo to the minus end of MTs. In mammals, two of its components are the very closely related light chains 1 (DYNLL1 also designated DLC8 because of its low MW about $8 \mathrm{kDa}$, or PIN) and 2, which are ubiquitous and among the most highly conserved proteins among species (see review by Pfister et al., 2006). A Blast search revealed a $95 \%$ amino acid sequence similarity with DNLL1 of Aedes aegypti and Culex quinquefasciatus and the "cut-up" protein of Drosophila melanogaster. The Drosophila homolog to DYNLL1 (Cdlc1, also known as ddlc1 and "cut-up") is ubiquitously expressed during development and in the adult and is required for both embryogenesis and cell differentiation (review by Pfister et al., 2006). In Rhodnius SG cells, the antibody against human DNLL1/2 recognized a small protein of about $15 \mathrm{kDa}$ on SDS-PAGE immunoblots that co-migrated with human DYNLL1, showing that Rhodnius possesses a peptide related to human DYNLL1.

We investigated the structural association between EcR and MTs in triple labeled experiments with the light chain dynein, DYNLL1 component of the dynein motor protein. We detected abundant dynein fluorescence in the cytoplasm of SG cells, substantial amounts of which co-localized with both EcR and with tubulin on MTs. Altogether, these findings imply that all four proteins EcR, Hsp90, FKBP52, and light chain dynein 1 are found together and with tubulin on MTs. Therefore, we suggest that EcR forms a heterocomplex with Hsp90 and FKBP52 that co-localizes on MTs with the motor protein dynein. These considerations suggest that the intracellular movement of EcR in Rhodnius cells employs the cytoskeletal tracks of MTs. The suggestion that MTs may use the EcR-Hsp90 heterocomplex as cargo for cytoplasmic transport has not been considered in any previous work. The relation of EcR with the MT network would also explain the present finding of EcR in axons of brain neurons in Rhodnius. Steroid receptors in axons have been detected in many mammalian systems (e.g., ER, Milner et al., 2005; AR, Milner et al., 2007). It is well-known that retrograde transport of cargo in axons requires cytoplasmic dynein and MTs (review by Hirokawa and Takemura, 2004).

There is a growing body of information, mostly derived from studies with the GR that supports the hypothesis that FKBP52 provides a link between the receptor-Hsp90-immunophilin heterocomplex and the cytoplasmic dynein motor complex (Harrell et al., 2004). For example, FKBP52 is required for dynein/dynactindependent retrograde movement of GR (Harrell et al., 2004), MR (Piwien Pilipuk et al., 2007; Galigniana et al., 2010), aryl hydrocarbon (Kazlauskas et al., 2001), p53 (Galigniana et al., 2004), and possibly estrogen receptor (Rayala et al., 2005). Interestingly, GR heterocomplexes isolated from the cytosol also contained tubulin, indicating that the dynein motor links the receptor heterocomplex to MTs (Harrell et al., 2004).
The above suggestions that EcR forms a complex with both Hsp90 and FKBP52 that attaches itself to the motor protein dynein on MTs for transport to the nucleus provides a mechanism by which EcR could exert its genomic effects. The complex seems to dissociate inside the nucleus. Therefore, we infer that the daily nucleocytoplasmic shuttling of EcR that occurs during defined periods of larval-adult development of Rhodnius employs cytoskeletal tracks for movement. Nucleocytoplasmic shuttling of EcR is further supported by the fact that EcRs in other species possess several nuclear export signals in addition to a nuclear import signal (Gwózdz et al., 2007; Betanska et al., 2011). Because the nuclear EcR rhythm is synchronous to the robust circadian rhythm of circulating ecdysteroids it is inferred that Rhodnius cells detect and respond to the daily signals in the rhythmic ecdysteroid titer.

These inferences raise some interesting questions. Is EcR transported to the nucleus alone or in the form of a heterodimer with Usp? It has been shown that EcR binds to ecdysteroids in the absence of its heterodimerization partner Usp (Grebe et al., 2000; Nieva et al., 2007) and EcR import to the nucleus increased in a hormone-dependent manner (Lammerding-Köppel et al., 1998; Nieva et al., 2007). Further, in a mammalian cell line which expresses either EcR or Usp, tagging of the proteins with the yellow fluorescent protein showed that Usp resides almost exclusively in the nucleus whereas EcR is found in both the nuclear and cytoplasmic compartments (Nieva et al., 2005; Gwózdz et al., 2007). Indeed, Usp possesses only nuclear localization signals but no nuclear export signals, whereas EcR possesses both (Gwózdz et al., 2007). Therefore, one view that we cannot exclude is the possibility that EcR in Rhodnius shuttles undimerized to the nucleus. Indeed, EcR undimerized with Usp induces transcription of glue genes in the SGs of Drosophila (Constantino et al., 2008). However, when both Usp and EcR are co-expressed in the above mammalian cell line, the presence of Usp favors nuclear localization of EcR (Gwózdz et al., 2007). Further, stable residency of EcR in the nucleus requires the formation of a heterodimer with Usp (Nieva et al., 2005). These findings support the opposite view that because EcR in Rhodnius cells resides in nuclei for many hours every night, it might require partnership with Usp in the nucleus. Such an arrangement would imply that Usp transport to the nucleus is also rhythmic in Rhodnius.

\section{ASSOCIATION OF ECR WITH THE MITOCHONDRIA}

A substantial amount of cytoplasmic EcR fluorescence does not co-localize with MTs. When we examined the association of EcR with mitochondria in double label experiments with a vital fluorescent dye that labels mitochondria, we detected substantial co-localization. Mitochondria are visible as punctate arrays of dots and rods that in many cases form dense aggregates, particularly in the perinuclear region. This perinuclear aggregation of mitochondria is a well-known distribution pattern of mitochondria within cells. The distribution pattern of EcR fluorescence in this area is similar to that of other nuclear receptors in mammalian cells (e.g., for GR, Scheller et al., 2000; for ER, Chen et al., 2004). Association of steroid receptors with mitochondria has been documented for many mammalian steroid receptors (references in Vafopoulou, 2009), where they are thought to influence mitochondrial gene activity. Indeed, hormone response elements in mitochondrial 
DNA and active hormone receptors were found to be imported into mitochondria and enhance the transcription of mitochondrial genes (references in Vafopoulou, 2009). The association of EcR with mitochondria of several cell types in Rhodnius raises the possibility that EcR, like its mammalian counterparts, may be involved in the coordination of ecdysteroid-responsive genes in mitochondria. The present finding of EcR along nerve axons highly remote from the nucleus (as seen for other steroid hormones; see above), emphasizes the generality of such non-genomic functions of steroid hormones.

We did not find much association of EcR with acidic organelles in cells such as lysosomes. The absence of EcR from acidic organelles suggests that lysosomes may not be involved in the

\section{REFERENCES}

Akner, G., Mossberg, K., Wikstrom, A. C., Sundqvist, K. G., and Gustafsson, J. A. (1991). Evidence for colocalization of glucocorticoid receptor with cytoplasmic microtubules in human gingival fibroblasts, using two different monoclonal anti-GR antibodies, confocal laser scanning microscopy and image analysis. J. Steroid Biochem. Mol. Biol. 39, 419-432.

Akner, G., Wikström, A. C., and Gustafsson, J. A. (1995). Subcellular distribution of the glucocorticoid receptor and evidence for its association with microtubules. J. Steroid Biochem. Mol. Biol. 52, 1-6.

Ampleford, E. J., and Steel, C. G. H. (1985). Circadian control of a daily rhythm in hemolymph ecdysteroid titer in the insect Rhodnius prolixus (Hemiptera). Gen. Comp. Endocrinol. 59, 453-459.

Arbeitman, M. N., and Hogness, D. S. (2000). Molecular chaperones activate the Drosophila ecdysone receptor, an RXR heterodimer. Cell 101, 67-77.

Azuma, K., Horie, K., Inoue, S., Ouchi, Y., and Sakai, R. (2004). Analysis of estrogen receptor $\alpha$ signalling complex at the plasma membrane. FEBS Lett. 577, 339-344.

Barsony, J., Renyi, I., and McKoy, W. (1997). Subcellular distribution of normal and mutant vitamin D receptors in living cells. J. Biol. Chem. 272, 5774-5782.

Betanska, K., Hönl, G., Spindler-Barth, M., and Spindler, K.-D. (2011). The importance of exportin and Ran for nucleocytoplasmic shuttling of the ecdysteroid receptor. Arch. Insect Biochem. Physiol. 76, 12-21.

Chambraud, B., Belabes, H., FontaineLenoir, V., Fellous, A., and Baulieu, E. E. (2007). The immunophilin FKBP52 specifically binds to tubulin and prevents microtubule formation. FASEB J. 21, 2787-2797.
Chen, J. Q., Delannoy, M., Cooke, C., and Yager, J. D. (2004). Mitochondrial localization of ERa and ERb in human MCF7 cells. Am. J. Physiol. Endocrinol. Metab. 286, E1011-E1022.

Constantino, B. F. B., Bricker, D. K., Alexandre, K., Shen, K., Merrian, J. R., Antoniewski, C., Callender, J. L., Henrich, V. C., and Andres, A. J. (2008). A novel ecdysone receptor mediates steroid-regulated developmental events during the mid-third instar of Drosophila. PLoS Genet. 4, e1000102. doi: 10.1371/journal.pgen.1000102

Dick, T., Krishanu, R., Salz, H. K., and Chia, W. (1996). Cytoplasmic dynein (ddlc1) mutations cause morphogenetic defects and apoptotic cell death in Drosophila melanogaster. Mol. Cell. Biol. 16, 1966-1977.

Dvorák, Z., Modrianský, M, Ulrichová, J., and Maurel, P. (2004). Speculations of the role of the microtubule network in glucocorticoid receptor signaling. Cell Biol. Toxicol. 20, 333-343.

Dvorák, Z., Modrianský, M, Ulrichová, J., Maurel, P., Vilarem, M. J., and Pascussi, J. M. (2005). Disruption of microtubules leads to glucocorticoid receptor degradation in $\mathrm{HeLa}$ cell line. Cell. Signal. 17, 187-196.

Dvorák, Z., Vrzal, R., Ulrichová, J., Macejová, D., Ondková, S., and Brtko, J. (2007). Expression, protein stability and transcriptional activity of retinoic acid receptors are affected by microtubules interfering agents and all-trans-retinoic acid in primary rat hepatocytes. Mol. Cell. Endocrinol. 267, 89-96.

Echeverría, P. C., Mazaira, G., Erlejman, A., Gomez-Sanchez, C., Piwien Pilipuk, G., and Galigniana, M. D. (2009). Nuclear import of the glucocorticoid receptor-Hsp90 complex is mediated by its interaction with through the nuclear pore complex

removal of EcR from the cell. This is unlike estrogen receptor $\alpha$ presence in lysosomes of mammalian cells, which suggested a role for lysosomes in receptor degradation as it shuttles out of the nucleus (Qualmann et al., 2000).

Since EcR exhibits a daily rhythm in Rhodnius that is driven by the rhythm in circulating steroid hormone levels (Vafopoulou and Steel, 2006; review by Steel and Vafopoulou, 2006), it is likely that both genomic and non-genomic effects will show synchronous daily rhythms.

\section{ACKNOWLEDGMENTS}

This work is supported by Natural Sciences Research Council of Canada, Discovery Grant 6669.

Nup62 and importin $\beta$. Mol. Cell. Biol. 29, 4788-4797.

Echeverria, P. C., and Picard, D. (2010). Molecular chaperones, essential partners of steroid hormone receptors for activity and mobility. Biochim. Biophys. Acto 1803, 641-649.

Elmogy, M., Iwami, M., and Sakurai, S. (2004). Presence of membrane ecdysone receptor in the anterior silk gland of the silkworm Bombyx mori. Eur. J. Biochem. 271, 3171-3179.

Elmogy, M., Iwami, M., and Sakurai, S. (2007). Solubilization of the ecdysone binding protein from anterior silk gland cell membranes of the silkworm, Bombyx mori. Zool. Sci. 24, 971-977.

Fujiwara, H., Jindra, M., Newitt, R., Palli, S. R., Hiruma, K., and Riddiford, L. M. (1995). Cloning of an ecdysone receptor homolog from Manduca sexta and the developmental profile of its mRNA in wings. Insect Biochem. Mol. Biol. 25, 845-856.

Galigniana, M. D., Erlejman, A. G., Monte, M., Gomez-Sanchez, C., and Piwien Pilipuk, G. (2010). The hsp90-FKBP52 complex links the mineralocorticoid receptor to motor proteins and persists bound to the receptor in early nuclear events. $\mathrm{Mol}$. Cell. Biol. 30, 1285-1298.

Galigniana, M. D., Harrell, J. M., O'Hagen, H. M., Ljungman, M., and Pratt, W. B. (2004). Hsp90-binding immunophilins link p53 to dynein during p53 transport to the nucleus. J. Biol. Chem. 279, 22483-22489.

Galigniana, M. D., Radanyi, C., Renoir, J. M., Housley, P. R., and Pratt, W. B. (2001). Evidence that the peptidylpropyl isomerase domain of the hsp90-binding immunophilin FKBP52 is involved in both dynein interaction and glucocorticoid receptor movement to the nucleus. J. Biol. Chem. 276, 14884-14889.

Galigniana, M. D., Scruggs, L. J., Herrington, J., Welsh, M. J., Carter-Su,
C., Housley, P. R., and Pratt, W. B. (1998). Heat shock protein 90-dependent (geldanamycininhibited) movement of the glucocorticoid receptor through the cytoplasm to the nucleus requires intact cytoskeleton. Mol. Endocrinol. 12, 1903-1913.

Grebe, M., Rauch, P., and SpindlerBarth, M. (2000). Characterization of subclones of the epithelial cell line from Chironomus tentans resistant to the insecticide RH5992, a nonsteroidal moulting hormone agonist. Insect Biochem. Mol. Biol. 30, 591-600.

Gwózdz, T., Dutko-Gwózdz, J., Nieva, C., Betanska, K., Orlowski, M., Kowalska, A., Dobrucki, J., SpindlerBarth, M., Spindler, K.-D., and Ozyhar, A. (2007). EcR and USP, components of the ecdysteroid nuclear receptor complex, exhibit differential distribution of molecular determinants directing subcellular trafficking. Cell. Signal. 19, 490-503.

Harrell, J. M., Murphy, P. J., Morishima, Y., Chen, H., Mansfield, J. F., Galigniana, M. D., and Pratt, W. B. (2004). Evidence for glucocorticoid receptor transport on microtubules by dynein. J. Biol. Chem. 279, 54647-54654.

Henrich, V. C. (2009). “The ecdysteroid receptor," in Insect Development: Morphogenesis, Molting and Metamorphosis, ed. L. I. Gilbert (London, GR: Academic Press), 261-304.

Hirokawa, N., and Takemura, R. (2004). Molecular motors in neuronal development, intracellular transport and diseases. Curr. Opin. Neurobiol. 14, 564-573.

Jindra, M., Malone, F., Hiruma, K., and Riddiford, L. M. (1996). Developmental profiles and ecdysteroid regulation of the mRNAs for two ecdysone receptor isoforms in the epidermis and wings of the tobacco hornworm, Manduca sexta. Dev. Biol. 180, 258-272. 
Kazlauskas, A., Sundstrom, S., Poellinger, L., and Pongratz, I. (2001). The hsp90 chaperone complex regulates intracellular localization of the dioxin receptor. Mol. Cell. Biol. 21, 2594-2607.

Lammerding-Köppel, M., SpindlerBarth, M., Steiner, E., Lezzi, M., Drews, U., and Spindler, K.-D. (1998). Immunohistochemical localization of ecdysteroid receptor and ultraspiracle in the epithelial cell line from Chironomus thummi (Insecta, Diptera). Tissue Cell 30, 187-194.

Manavathi, B., Acconsia, F., Rayala, S. K., and Kumar, R. (2006). An inherent role of microtubule network in the action of nuclear receptor. Proc. Natl. Acad. Sci. U.S.A. 103, 15981-15986.

Milner, T. A., Ayoola, K., Drake, C. T., Herrick, S. P., Tabori, N. E., and McEwen, B. S. (2005). Ultrastructural localization of estrogen receptor $\beta$ immunoreactivity in the rat hippocampal formation. J. Comp. Neurol. 491, 81-95.

Milner, T. A., Hernandez, F. J., Herrick, S. P., Pierce, J. P., Iadecola, C., and Drake, C. T. (2007). Cellular and subcellular localization of androgen receptor immunoreactivity relative to $\mathrm{Cl}$ adrenergic neurons in the rostral ventrolateral medulla of male and female rats. Synapse 61, 268-278.

Nakagawa, Y., and Henrich, V. C. (2009). Arthropod nuclear receptors and their role in molting. FEBS J. 276, 6128-6157.

Nieva, C., Gwózdz, T., Dutko-Gwózdz, J., Wiedenmann, J., Spindler-Barth, M., Wieczorek, E., Dobrucki, J., Dus, D., Henrich, V., Ozyhar, A., and Spindler, K.-D. (2005). Ultraspiracle promotes the nuclear localization of ecdysteroid receptor in mammalian cells. Biol. Chem. 386, 463-470.

Nieva, C., Spindler-Barth, M., Azoitei, A., and Spindler, K.-D. (2007). Influence of hormone intracellular localization of the Drosophila melanogaster ecdysteroid receptor (EcR). Cell. Signal. 19, 2582-2587.

Nollen, E. A., and Morimoto, R. I. (2002). Chaperoning signalling pathways: molecular chaperones as stress-sensing 'heat shock' proteins. J. Cell. Sci. 115, 2809-2816.
Ou, Q., Magico, A., and King-Jones, K. (2012). Nuclear receptor DHR4 controls the timing of steroid hormone pulses during Drosophila development. PLoS Biol. 9, e1001160. doi: 10.1371/journal.pbio. 1001160

Perrot-Applanat, M., Cibert, C., Géraud, G., Renoir, J.-M., and Baulieu, E. E. (1995). The 59 kDa FK506-binding protein, a $90 \mathrm{kDa}$ heat shock protein binding immunophilin (FKBP59$\mathrm{HBI}$ ), is associated with the nucleus, the cytoskeleton and mitotic apparatus. J. Cell. Sci. 108, 2037-2051.

Pfister, K. K., Shah, P. R., Hummerich, H., Russ, A., Cotton, J., Annuar, A. A., King, S. M., and Fisher, E. M. C. (2006). Genetic analysis of the cytoplasmic dynein subunit families. PLoS Genet. 2, 11-26. doi: 10.1371/journal.pgen.0020001

Picard, D. (2006). Chaperoning steroid hormone action. Trends Endocrinol. Metab. 17, 230-236.

Piwien Pilipuk, G., Vinson, G. P., Sanchez, C. G., and Galigniana, M. D. (2007). Evidence for NL1independent nuclear translocation of the mineralocorticoid receptor. Biochem. 46, 1389-1397.

Pratt, W. B., Galigniana, M. D., Harrell, J. M., and DeFranco, D. B. (2004a) Role of hsp90 and the hsp90-binding immunophilins in signalling protein movement. Cell. Signal. 16, 857-872.

Pratt, W. B., Galigniana, M. D., Morishima, Y., and Murphy, P. J. (2004b). Role of molecular chaperones in steroid receptor action. Essays Biochem. 40, 41-58.

Pratt, W. B., and Toft, D. O. (2003). Regulation of signal protein function and trafficking by the hsp90/hsp70based chaperone machinery. Exp. Biol. Med. 228, 111-133.

Qualmann, B., Kessels, M. M., Thole, H. H., and Sierralta, W. D. (2000). A hormone pulse influences distributions and leads to lysosomal accumulation of the estradiol receptor a in target tissues. Eur. J. Cell Biol. 79, 383-393.

Ratajczak, T., Ward, B. K., and Minchin, R. F. (2003). Immunophilin chaperones in steroid receptor signalling. Curr. Top. Med. Chem. 3, 1348-1357.

Rayala, S. K., den Hollander, P., Balasenthil, S., Yang, Z., and Broaddus, R. R. (2005). Functional regulation of oestrogen receptor pathway by the dynein light chain 1. EMBO Rep. 6 , 538-545.
Riddiford, L. M., Hiruma, K., Zhou, X. F., and Nelson, C. A. (2003). Insights into the molecular basis of the hormonal control of molting and metamorphosis from Manduca sexta and Drosophila melanogaster. Insect Biochem. Mol. Biol. 33, 1327-1338.

Scheller, K., Sekeris, C. E., Krohne, G. Hock, R., Hansen, I. A., and Scheer, U. (2000). Localization of glucocorticoid hormone receptors in mitochondria of human cells. Eur. J. Cel Biol. 79, 299-307.

Schlattner, U., Vafopoulou, X., Steel, C. G. H., Hormann, R. E., and Lezzi, M. (2006). Non-genomic ecdysone effects and the invertebrate nuclear steroid hormone receptor EcR -new role for an "old" receptor? Mol. Cell. Endocrinol. 247, 64-72.

Shimazu, K. K. (2003). Expression and intracellular localization of progesterone receptors in cultured human gingival fibroblasts. J. Periodont. Res. 38, 242-246.

Shu, Y., Du, Y., and Wang, J. (2011). Molecular characterization and expression patterns of Spodoptera litura heat shock protein $70 / 90$ and their response to zinc stress. Comp. Biochem. Physiol. A Mol. Integr. Physiol. 158, 102-110.

Song, Q., Alnemri, E. S., Litwack, G., and Gilbert, L. I. (1997). An immunophilin is a component of the insect ecdysone receptor (EcR) complex. Insect Biochem. Mol. Biol. 27, 973-982.

Spindler, K.-D., Hönl, C., Tremmel, C. H., Braun, S., Ruff, H., and SpindlerBarth, M. (2009). Ecdysteroid hormone action. Cell. Mol. Life Sci. 66 , 3837-3850.

Steel, C. G. H., and Vafopoulou, X. (2006). Circadian orchestration of developmental hormones in the insect, Rhodnius prolixus. Comp. Biochem. Physiol. A Mol. Integr. Physiol. 144, 351-364.

Vafopoulou, X. (2009). Ecdysteroid receptor $(\mathrm{EcR})$ is associated with microtubules and with mitochondria in the cytoplasm of prothoracic gland cells of Rhodnius prolixus (Hemiptera). Arch. Insect Biochem. Physiol. 72, 249-262.

Vafopoulou, X., and Steel, C. G. H. (1991). Circadian regulation of ecdysteroid synthesis in prothoracic glands of the insect, Rhodnius prolixus (Hemiptera). Gen. Comp. Endocrinol. 83, 1-9.
Vafopoulou, X., and Steel, C. G H. (2006). Ecdysteroid hormone nuclear receptor (EcR) exhibits circadian cycling in certain tissues, but not others, during development in Rhodnius prolixus (Hemiptera). Cell Tissue Res. 323, 443-455.

Vafopoulou, X., Steel, C. G. H., and Terry, K. (2005). Ecdysteroid receptor (EcR) shows marked differences in temporal patterns between tissues during larval-adult development in Rhodnius prolixus: correlations with haemolymph ecdysteroid titres. J. Insect Physiol. 51, 27-38.

Welte, M. A. (2004). Bidirectional transport along microtubules. Curr. Biol. 14, R525-R537.

Wigglesworth, V. B. (1985). "Historical perspectives," in Comprehensive Insect Physiology, Biochemistry and Pharmacology. Vol. 7 Endocrinol$\operatorname{ogy} I$, eds G. A. Kerkut and L. I. Gilbert (Oxford, GR: Pergamon Press), 1-24.

Zhang, Q., and Denlinger, D. L. (2010). Molecular cloning of heat shock protein 90,70 and 70 cognate cDNAs and their expression patterns during thermal stress and pupal diapause in the corn earworm. J. Insect Physiol. $56,138-150$.

Conflict of Interest Statement: The authors declare that the research was conducted in the absence of any commercial or financial relationships that could be construed as a potential conflict of interest.

Received: 09 October 2011; accepted: 06 March 2012; published online: 23 March 2012.

Citation: Vafopoulou $X$ and Steel CGH (2012) Cytoplasmic travels of the ecdysteroid receptor in target cells: pathways for both genomic and non-genomic actions. Front. Endocrin. 3:43. doi: 10.3389/fendo.2012.00043

This article was submitted to Frontiers in Experimental Endocrinology, a specialty of Frontiers in Endocrinology.

Copyright (C) 2012 Vafopoulou and Steel. This is an open-access article distributed under the terms of the Creative Commons Attribution Non Commercial License, which permits non-commercial use, distribution, and reproduction in other forums, provided the original authors and source are credited. 